\title{
Exosomes mediate sensory hair cell protection in the inner ear
}

\author{
Andrew M. Breglio, $, 1,2,3,4$ Lindsey A. May, ${ }^{1}$ Melanie Barzik, ${ }^{1}$ Nora C. Welsh, ${ }^{1}$ Shimon P. Francis, ${ }^{1}$ Tucker Q. Costain, ${ }^{1}$ Lizhen Wang, ${ }^{1}$ \\ D. Eric Anderson, ${ }^{5}$ Ronald S. Petralia, ${ }^{1}$ Ya-Xian Wang, ${ }^{1}$ Thomas B. Friedman, ${ }^{1}$ Matthew J.A. Wood, ${ }^{2,6}$ and Lisa L. Cunningham ${ }^{1}$ \\ ${ }^{1}$ National Institute on Deafness and Other Communication Disorders (NIDCD), NIH, Bethesda, Maryland, USA. ${ }^{2}$ Department of Physiology, Anatomy and Genetics, University of Oxford, Oxford, \\ United Kingdom. ${ }^{3}$ Icahn School of Medicine at Mount Sinai, New York, New York, USA. ${ }^{4}$ NIH Oxford-Cambridge Scholars Program, Bethesda, Maryland, USA. ${ }^{5}$ National Institute of Diabetes and \\ Digestive and Kidney Diseases (NIDDK), NIH, Bethesda, Maryland, USA. ㅁDepartment of Paediatrics, University of Oxford, Oxford, United Kingdom.
}

Hair cells, the mechanosensory receptors of the inner ear, are responsible for hearing and balance. Hair cell death and consequent hearing loss are common results of treatment with ototoxic drugs, including the widely used aminoglycoside antibiotics. Induction of heat shock proteins (HSPs) confers protection against aminoglycoside-induced hair cell death via paracrine signaling that requires extracellular heat shock 70-kDa protein (HSP70). We investigated the mechanisms underlying this non-cell-autonomous protective signaling in the inner ear. In response to heat stress, inner ear tissue releases exosomes that carry HSP7O in addition to canonical exosome markers and other proteins. Isolated exosomes from heatshocked utricles were sufficient to improve survival of hair cells exposed to the aminoglycoside antibiotic neomycin, whereas inhibition or depletion of exosomes from the extracellular environment abolished the protective effect of heat shock. Hair cell-specific expression of the known HSP70 receptor TLR4 was required for the protective effect of exosomes, and exosomal HSP70 interacted with TLR4 on hair cells. Our results indicate that exosomes are a previously undescribed mechanism of intercellular communication in the inner ear that can mediate nonautonomous hair cell survival. Exosomes may hold potential as nanocarriers for delivery of therapeutics against hearing loss.

\section{Introduction}

Disabling hearing loss affects approximately $6.1 \%$ of the global population (1). Most hearing loss is due to death of sensory hair cells of the inner ear. These mechanosensitive receptor cells mediate hearing and balance functions in the cochlea and vestibular organs, respectively. A variety of stresses can kill hair cells, including aging, noise trauma, and clinical treatment with platinum-based cancer chemotherapeutics or aminoglycoside antibiotics, so-called ototoxic drugs. Ototoxic drugs cause permanent hearing loss in approximately 500,00 people annually in the US alone (2). In patients treated with aminoglycoside antibiotics, hearing loss is caused by direct hair cell damage. Aminoglycosides enter hair cells via both hair cellspecific membrane channels and endocytosis, resulting in intracellular accumulation and subsequent cytotoxicity (3-7). Activation of the heat stress response improves hair cell survival and preserves hearing in animal models of ototoxicity $(2,8-11)$. Elucidation of the mechanisms underlying this protective effect will advance both the basic science of cochlear function and the clinical development of therapies to prevent or reverse hearing loss.

\section{Related Commentary: p. 2206}

Authorship note: AMB, LAM, and MB contributed equally to this work. Conflict of interest: Matthew Wood is a founder and director of Evox Therapeutics. Copyright: () 2020, American Society for Clinical Investigation. Submitted: March 14, 2019; Accepted: January 30, 2020; Published: April 20, 2020. Reference information: J Clin Invest. 2020;130(5):2657-2672. https://doi.org/10.1172/JCl128867.
Sensory hair cells are surrounded by glia-like supporting cells, named for their ability to support normal hair cell function. Supporting cells can mediate both the death and survival of hair cells (12-18). Our previous work demonstrated that supporting cells promote hair cell survival through paracrine signaling that requires extracellular heat shock 70-kDa protein (HSP70) (18). Here, we tested the hypothesis that HSP70-dependent paracrine protection of hair cells is mediated by exosomes. Studies in other systems have demonstrated extracellular release of HSP70 via incorporation into exosomes $(19,20)$ and frequently identified HSP70 as a protein component of purified exosomes (21). Exosomes are small (50- to 150-nm) extracellular vesicles (EVs) that are released from most cell types and can mediate intercellular communication via receptor signaling or cargo delivery to recipient cells (22). Our data indicate a crucial function for HSP70-containing exosomes in the inner ear stress response and in mediating non-cell-autonomous protection of hair cells.

\section{Results}

HSP7O is induced in glia-like supporting cells after heat shock. Activation of the heat stress response in the inner ear protects sensory hair cells from ototoxic drug exposure $(2,8-11)$, and this pro-survival effect is mediated through paracrine signaling that requires HSP70 (18). Using whole organ cultures of utricles (a vestibular organ) from adult mice, we previously demonstrated that heat shock results in HSP7O upregulation in glia-like supporting cells with negligible induction in sensory hair cells (18). Here, we confirmed induction of HSP7O in supporting cells after heat shock using additional Abs and improved imaging methods. Utricles 

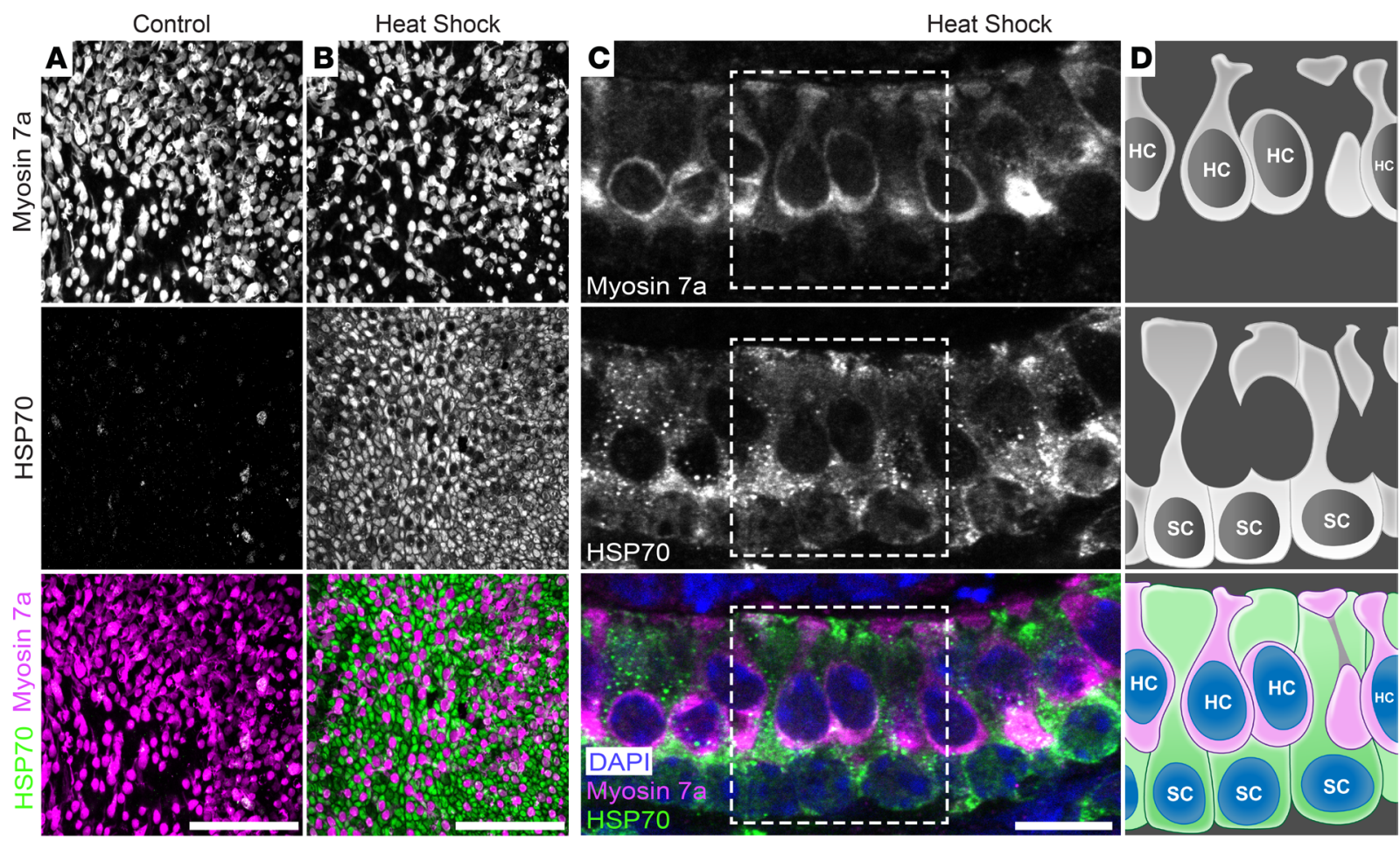

Figure 1. HSP70 is upregulated in supporting cells upon heat shock. Mouse utricles were cultured under control or heat shock conditions, fixed 6 hours later, and labeled with Abs against myosin 7a (hair cell marker, magenta) and inducible HSP70 (green). (A and B) Confocal images of whole-mount utricles show sensory hair cells surrounded by glia-like supporting cells. (A) Inducible HSP7O was not detected under control culture conditions. (B) Heat shock induced HSP70 specifically in supporting cells, with very little induction in hair cells. (C) Cryosection of a heat-shocked utricle confirmed negligible induction of HSP70 (green) in hair cells (labeled with myosin 7a, magenta), whereas HSP70 was substantially upregulated in supporting cells. Nuclei in A-C were stained with DAPI (blue). (D) Schematics showing locations of hair cells (HC, magenta) and supporting cells (SC, green) in boxes outlined in C, with nuclei indicated in blue in the lower right panel. Scale bars: $50 \mu \mathrm{m}$ (A and $\mathbf{B}$ ) and $10 \mu \mathrm{m}$ (C).

were heat shocked at $43^{\circ} \mathrm{C}$ for 30 minutes and upregulation of the inducible forms of HSP7O (HSPA1A and HSPA1B) was assessed 6 hours later (Figure 1). We found that HSP70 was induced in supporting cells with little to no induction in hair cells in both wholemount (Figure 1, A and B) and cryosectioned (Figure 1C) utricles after heat shock. These results are consistent with our previous study (18), which also demonstrated that HSP70 is secreted from heat-shocked utricles. Importantly, this extracellular HSP70 was protective against hair cell death caused by the ototoxic antibiotic neomycin, confirming that HSP70 acts non-cell autonomously.

Heat shock induces the release of exosomes from inner ear tissue. HSP7O is secreted from many cell types via exosomes (18, 23-27). To determine whether HSP70-dependent intercellular communication in the inner ear is facilitated by exosomes, we explored the relationship between heat stress and EV release. Utricles were cultured in serum-free media for 24 hours, and the utricle-conditioned media were analyzed for exosome-sized particles by nanoparticle tracking analysis (NTA), a method for real-time quantification of particles in liquids (ref. 28 and Figure 2A). Conditioned media from control utricles contained exosome-sized $(\sim 50-150 \mathrm{~nm})$ particles. Heat shock increased exosome release by 2.38-fold (95\% CI $=1.74-2.82$ ) (Figure 2A).

Exosomes released from heat-shocked utricles were isolated using an established procedure of differential ultracentrifugation (refs. 29, 30 and schematized in Figure 2B) and visualized by transmission electron microscopy (TEM). Consistent with previously described exosomes from other systems $(31,32)$, TEM analysis of the final exosome-containing fraction showed small vesicles (90- to 100-nm diameter, 40 - to $200-\mathrm{nm}$ range) with a cup-shaped morphology (Figure 2C). Together, these results indicate that control utricles constitutively release exosomes and that heat shock substantially increases exosome release.

Identification of proteins in utricle-derived exosomes. Exosomes can carry a variety of cargo, such as proteins (including HSP70), lipids, and nucleic acids (21). We used tandem mass spectrometry to identify proteins associated with exosomes released from heat-shocked utricles. To yield high-purity samples, exosomes were separated from soluble proteins and protein complexes in utricle-conditioned media by ultrafiltration followed by size exclusion chromatography (33). We analyzed chromatographic fractions containing vesicles as the "exosome" sample and the remaining fractions as the "non-exosomal fraction" sample (analogous to the supernatant resulting from purification of exosomes by ultracentrifugation). We identified 291 protein families in exosomes, only 56 of which were also present in the non-exosomal fraction (Figure 3A). A Mascot database search using the peptides that were exclusively detected in the exosome sample identified several proteins commonly enriched in exosomes (Table 1), confirming that the utricle-derived vesicles were exosomes. These established exosomal markers included tetraspanin proteins (CD9, CD63, and CD81), as well as tumor susceptibility 
A

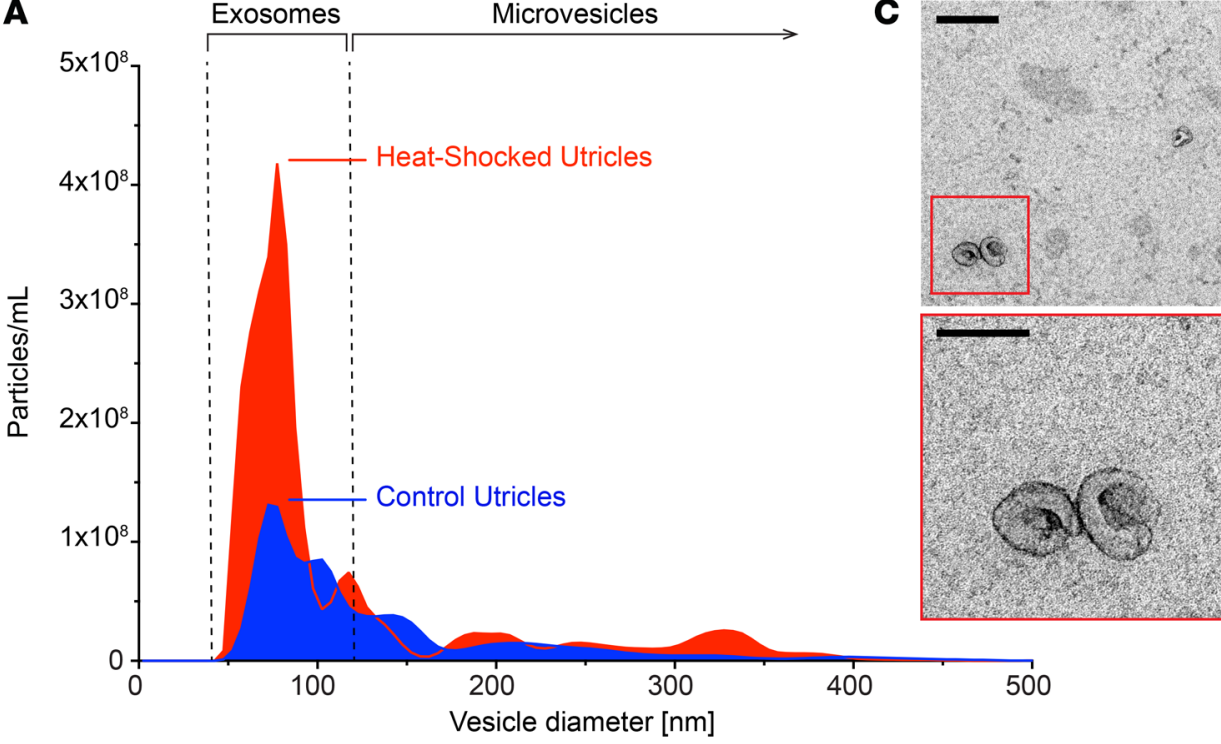

B

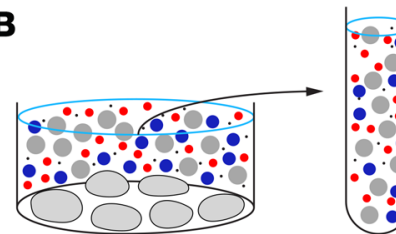

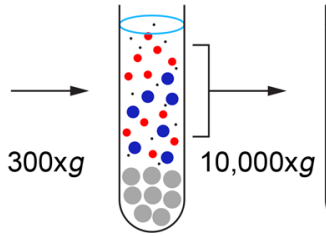

Tissue Debris

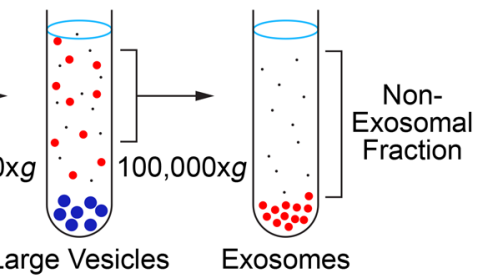

Figure 2. Heat stress stimulates exosome release from inner ear tissue.

(A) Nanoparticle tracking analysis of conditioned media from utricles cultured under control conditions shows release of exosome-sized ( $50-150 \mathrm{~nm}$ diameter) particles from control utricles. Heat shock resulted in a 2.4-fold increase in exosome release. Horizontal bars denote typical size ranges of exosomes and microvesicles. (B) Schematic of differential ultracentrifugation procedure used to isolate exosomes from utricle-conditioned culture medium. This process sequentially sediments extracellular components of decreasing size (tissue debris, gray; large vesicles, blue), with exosomes (red) isolated in the final pellet. (C) Isolated exosomes from utricle-conditioned media visualized by TEM were approximately $90 \mathrm{~nm}$ in diameter and displayed canonical cup-shaped morphology. Scale bars: $200 \mathrm{~nm}$ (top); 100 $\mathrm{nm}$ (enlarged inset). gene 101 (TSG101), a member of the endosomal sorting complex required for transport I (ESCRT-I) complex, and programmed cell death 6-interacting protein/ALG-2-interacting protein X (ALIX), an ESCRT accessory protein (reviewed in ref. 34). Notably, the peptide matches for members of the HSP70 family were more abundant in the exosome fraction than in the non-exosomal fraction, suggesting that HSP70 family proteins, including the inducible forms (HSPA1A/ HSPA1B), may be specifically enriched in exosomes.

We performed Gene Ontology (GO) enrichment analysis on proteins identified in utricle-derived exosomes using the Protein Analysis Through Evolutionary Relationships (PANTHER) classification system (version 13.1) $(35,36)$, followed by removal of redundant ontology terms using Reduce and Visualize Gene Ontology (REVIGO) (37). Analysis by cellular component classes identified enrichment of 35 GO terms, with the most statistically significant being "extracellular exosomes" (Figure 3B, GO:0070062). Analysis by biological process identified enrichment of $69 \mathrm{GO}$ terms, with terms associated with inner ear tissue ("sensory perception of sound," GO:0007605; "sensory perception of mechanical stimulus," GO:0050954) being among the top 5 statistically significant results (Figure 3C).

The integrin family of transmembrane adhesion receptors control various fundamental cellular functions, including proliferation and cell survival $(38,39)$. Integrins are heterodimers composed of $\alpha$ and $\beta$ subunits. In exosomes, integrins are embedded in the membrane, where they mediate intercellular communication. Specifically, the complement of exosomal integrins specifies the target organs or cell types with which an exosome interacts (40). Three $\alpha$ subunits $(\alpha-3, \alpha-7$, and $\alpha-V)$ and $1 \beta$ subunit ( $\beta-1)$ were identified in exosomes from utricles, suggesting that they may specify exosomal tropism in the inner ear (Table 2).
Isolated exosomes improve hair cell survival. We next investigated whether the HSP70-carrying exosomes are protective against aminoglycoside-induced hair cell death. Cultured utricles were heat shocked and exosomes purified from conditioned media via differential ultracentrifugation (Figure 4A). Both the pelleted exosome fraction and the non-exosomal fraction (containing extracellular, soluble proteins and nucleic acids not associated with exosomes) were tested for their ability to protect hair cells from ototoxicity. Treatment of recipient utricles with the aminoglycoside antibiotic neomycin killed approximately $48 \%$ of hair cells (Figure 4, B and C). Application of utricle-derived exosomes significantly reduced neomycin-induced hair cell death, whereas the non-exosomal (supernatant) fraction was not protective (Figure 4, $\mathrm{B}$ and $\mathrm{C}$ ). Thus, utricle-derived exosomes are protective against aminoglycoside-induced hair cell death.

Exosome biogenesis is required for the protective effect of heat shock. We assessed the necessity of exosomes for the protective effect of heat stress through pharmacological inhibition of exosome biogenesis. Exosomes are generated as intraluminal vesicles that bud into a multivesicular body, a process that requires the sphingolipid ceramide (Figure 5A and ref. 41). Spiroepoxide is a selective and irreversible inhibitor of neutral sphingomyelinase II (N-SMase), the enzyme that catalyzes cellular production of ceramide $(42,43)$. NTA of conditioned media revealed that spiroepoxide treatment resulted in a significant decrease in the number of exosomes released by heat-shocked utricles $\left(0.5 \times 10^{9}\right.$ vs. $1.5 \times 10^{9}$ exosomes/mL) (Figure 5B). Importantly, neither heat shock alone nor heat shock in the presence of spiroepoxide resulted in hair cell death relative to control utricles (Figure 5C). Therefore, the reduced quantity of exosomes released from spiroepoxide-treated 
A

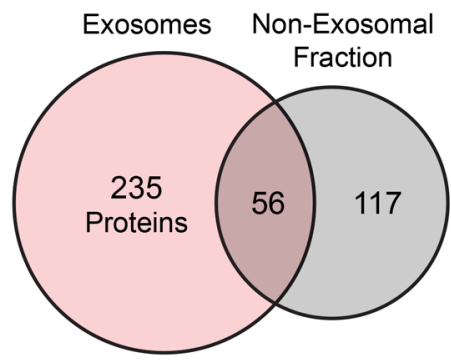

B

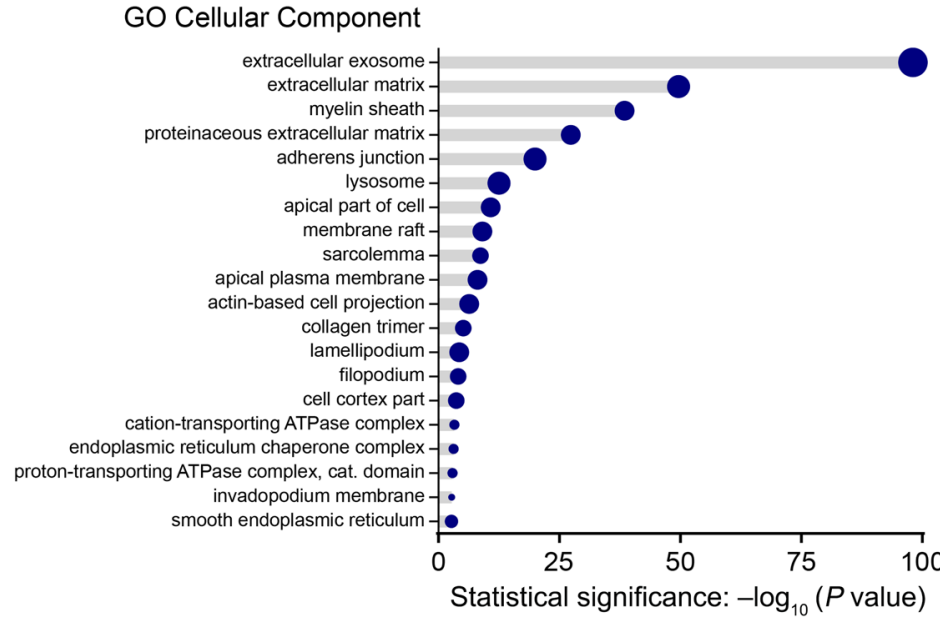

C

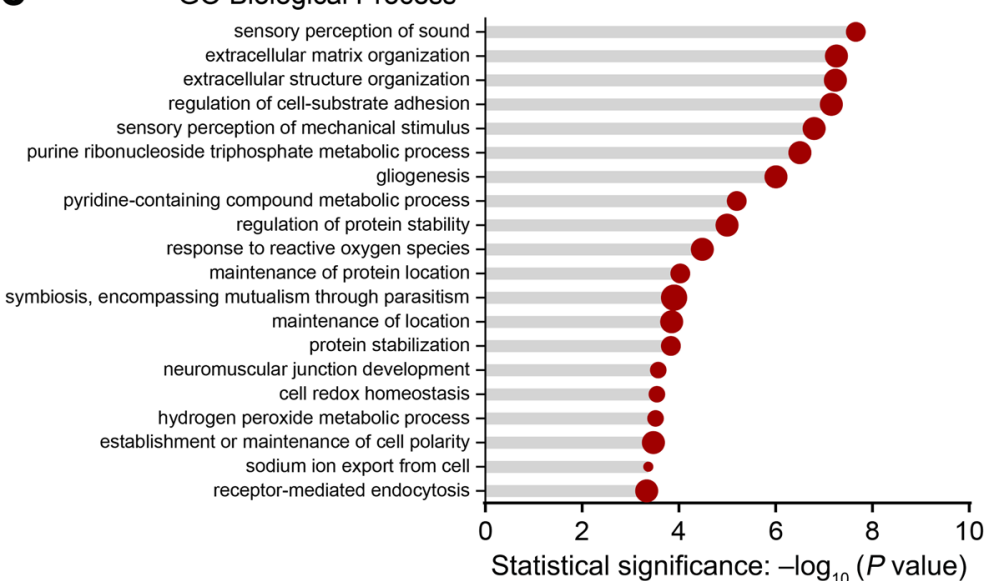

Figure 3. Proteins identified in utricle exosomes. Proteins associated with exosomes and non-exosomal proteins secreted from heat-shocked utricles were identified using tandem mass spectrometry. Exosomes were isolated from conditioned media via size-exclusion chromatography and analyzed in parallel with the exosome-depleted media (non-exosomal fraction). (A) A total of 291 unique protein families were identified in exosomes, 56 of which were also found in the non-exosomal fraction. (B) The 20 most significantly enriched GO cellular component terms for proteins identified in utricle-derived exosomes. (C) The 20 most significantly enriched biological process GO terms for proteins identified in exosomes from utricles.

types include macrophages, fibroblasts, and transitional epithelium. We used a differential fluorescence labeling approach to determine the contributions of hair cells and supporting cells to the total exosome population (Figure 6A). mTmG global double-fluorescent reporter mice express membrane-targeted (myristoylated) tandem dimer Tomato (mtdTomato) protein in all cells and myristoylated green fluorescent protein (mGFP) following Cre-mediated excision of mtdTomato (44). Myristoylation of both fluorophores resulted in their incorporation into lipid membranes, including exosome membranes. NTA of conditioned media from heatshocked mTmG utricles and utricles of age-matched control (WT) mice confirmed that lipid modification of the fluorophores did not interfere with exosome production or release (Figure 6B). mTmG mice were crossed with mice expressing Cre recombinase under control of the growth factor-independent 1 (Gfil) promoter to generate mice in which $96 \%$ of hair cells expressed mGFP, whereas all other cell types expressed mtdTomato (Figure 6C). We used an established microscopy-based technique $(45,46)$ to detect and quantify fluorescent exosomes. This method does not rely on resolving exosomes, which are below the resolution limit of a standard confocal microscope ( $200 \mathrm{~nm})$; instead, it allows detection of exosome-associated fluorescent proteins (mtdTomato or mGFP). The size of the fluorescent puncta (Airy disks) sampled on the microscope's photodetector is not equivalent to the size of the exosomes but instead is related to the fluorescence intensity of the utricles cannot be attributed to cell death. We next used spiroepoxide to determine whether exosome biogenesis is required for the pro-survival effect of heat shock in utricles. Neomycin killed approximately $40 \%$ of hair cells, whereas heat shock significantly $(P=0.020)$ improved hair cell survival. Notably, inhibition of exosome biogenesis using spiroepoxide abolished the protective effect of heat shock (Figure 5D). Thus, ceramide is necessary for the formation of utricular exosomes, and inhibition of ceramide production using spiroepoxide inhibits exosome release from cultured utricles. Together, these data demonstrate that the hair cell protection induced by heat stress in utricles requires exosomes.

Supporting cells produce more exosomes compared with hair cells. We next investigated the cellular source(s) of protective exosomes in the utricle. Utricles contain several cell types, predominantly sensory hair cells and glia-like supporting cells. Additional cell point source. Using this method, $17.4 \% \pm 1 . \%$ (mean \pm SEM) of exosomes purified from heat-shocked utricles from $\mathrm{mTmG}$ mice crossed with Gfil-Cre mice were identified as mGFP positive, indicating that they originated from hair cells (Figure 6, C-F). In the complementary experiment, $\mathrm{mTmG}$ mice were crossed with mice expressing tamoxifen-inducible Cre recombinase (CreER) under control of the glial high-affinity glutamate transporter (GLAST, Slc1a3) to produce mice in which $65 \%$ of supporting cells expressed mGFP, whereas all other cell types expressed mtdTomato (Figure $6 \mathrm{G})$. We found that $25.3 \% \pm 2.0 \%$ of exosomes isolated from these utricles after heat shock were mGFP positive (i.e., originated from supporting cells) (Figure 6, $\mathrm{H}-\mathrm{J}$ ). We adjusted for the efficiency of recombination by the 2 Cre lines ( $96 \%$ efficiency for Gfil-Cre; $65 \%$ efficiency for GLAST-CreER) and found that approximately $38 \%$ of utricle-derived exosomes originated from supporting cells, 


\section{Table 1. Common exosome markers}

$\begin{array}{lcc}\text { Protein (gene symbol) } & \text { Exosomes } & \begin{array}{c}\text { Non-exosomal } \\ \text { fraction }\end{array} \\ \text { CD9 antigen }(C d 9) & + & - \\ \text { CD81 antigen (Cd81) } & + & - \\ \text { CD63 antigen (Cd63) } & + & - \\ \text { Tumor susceptibility gene 101 (Tsg101) } & + & - \\ \text { Programmed cell death 6-interacting protein (Alix) } & + & - \\ \text { HSP70 protein family } & ++ & +\end{array}$

Proteins commonly used as exosome markers were confirmed in the utricle-derived exosome fraction but not in the non-exosomal fraction.

approximately $18 \%$ from hair cells, and approximately $44 \%$ from other cell types including macrophages, fibroblasts, and transitional epithelium (Figure 6K). These data demonstrate that both hair cells and supporting cells release exosomes, with supporting cells releasing about twice as many exosomes as hair cells.

HSP7O is required for the pro-survival effect of exosomes. We previously reported that extracellular HSP7O is required for the protective effect of heat shock against neomycin-induced hair cell death (18). Here, we show that (a) HSP70 was associated with utricle-derived exosomes (Table 1); (b) exosomes were essential for the protective response induced by heat stress (Figure 4, B and C, and Figure 5D); and (c) the non-exosomal fraction was not protective (Figure 4, B and C). Together, these data support the hypothesis that exosome-associated HSP7O is required for the heat shockinduced pro-survival effect.

To directly test this hypothesis, we first confirmed HSP70 distribution by Western blot analysis (Figure 7A; see complete unedited blots in the supplemental material, available online with this article; https://doi.org/10.1172/JCI128867DS1). Exosomes and the non-exosomal supernatant fraction were isolated from heat-shocked utricles by differential ultracentrifugation (Figure 2B), and the macromolecules in the non-exosomal fraction were further concentrated using a centrifugal filtration device $(3000$ molecular weight cutoff [MWCO]). Total protein concentrations for each sample were determined using a bicinchoninic acid (BCA) assay. We analyzed equivalent protein amounts by Western blotting and found that HSP7O and the canonical exosome marker CD81 were exclusively detected in exosomes and absent from the non-exosomal fraction. These data are in agreement with our proteomics analyses (Table 1) and indicate that HSP70 released from utricles is exosome associated.

We next used immunoelectron microscopy to visualize exosomal HSP70. Exosomes from heat-shocked utricles showed positive immunogold labeling for HSP70, with gold particles clustered around the exosome membrane (Figure 7, B and C). Control samples treated with only the gold-conjugated secondary Ab showed no HSP70 immunoreactivity (data not shown). These results confirm our proteomics and Western blot data demonstrating that utricle-derived exosomes carry HSP7O (Table 1), and they suggest that HSP7O is associated with the exosomal membrane, either directly by integration into the lipid bilayer or indirectly by formation of complexes with transmembrane proteins, as has been reported for HSP7O in exosomes from other systems $(26,47-50)$.
We used a function-blocking $\mathrm{Ab}(\mathrm{fbAb})$ against $\operatorname{HSP70}(51,52)$ to determine whether exosome-associated HSP7O is required for the protective effect of exosome application (Figure 7D). Utricles exposed to neomycin showed significant death of hair cells, which was again reduced by the application of isolated exosomes. This protective effect was abolished when isolated exosomes were pretreated with a fbAb against HSP70. The HSP70 fbAb alone had no effect on hair cell survival (Figure 7D). These data indicate that HSP70 is critical for the protective effect of exosomes and that HSP7O is at least partially exposed on the exosome surface, where it is accessible by the anti-HSP70 Ab.

To examine whether non-exosomal HSP70 is sufficient for protection, we applied soluble HSP70 to neomycin-treated utricles (Figure 7E). We performed a stringent quantification of hair cell density by manually counting surviving hair cells in the entire posterior region of treated utricles (average area: $80,000 \mu \mathrm{m}^{2}$ ). Although application of exosomes isolated from heat-shocked utricles again improved hair cell survival in neomycin-treated utricles, addition of exogenous HSP70 (i.e., HSP70 not associated with exosomes) was not protective (Figure 7E). These results indicate that association of HSP7O with exosomes is required to induce a pro-survival response in hair cells, and they imply that additional exosomal properties and/or cargo also contribute to the protective effect of exosome application.

Together, our data indicate that HSP70 is associated with the membrane of utricle-derived exosomes and that this exosomal HSP70 is required for the protective effect of exosomes against neomycin-induced hair cell death.

Exosomes interact with TLR 4 on hair cells. Extracellular HSP7O is an endogenous ligand of TLR4 (53-56). Binding of HSP70 to TLR4 mediates protection from ischemia/reperfusion injury in cardiomyocytes and from lethal hyperoxic lung injury (57-59). We therefore explored the role of TLR4 signaling in exosome-mediated hair cell protection in utricles exposed to ototoxic stress. To specifically investigate whether TLR4 is required in hair cells, B6(Cg)-Tlr $4^{\text {tml.1Karp }} / \mathrm{J}$ (TLR4-loxP) mice were crossed with B6.Cg-Tg(Atoh1-Cre)1Bfri/J mice expressing Cre recombinase under the control of atonal bHLH transcription factor 1 (Atoh1) (Atoh1-Cre) to produce offspring in which TLR4 was conditionally deleted from hair cells (TLR4-cKO mice). In utricles from littermate control mice [B6(Cg)Tlr $4^{\text {tml.1Karp }} \mathrm{XB6}$ 6.Cg; referred to herein as WT mice], the application of exosomes from heat-shocked utricles improved hair cell survival following neomycin exposure (Figure 8A), similar to our data from CBA/J mice (Figure 4, B and C). However, when applied to TLR4cKO utricles, exosomes did not improve hair cell survival in the presence of neomycin. These results indicate that exosome-mediated protection requires TLR4 expression in hair cells.

Table 2. Exosome-associated integrins

$\begin{array}{ll}\boldsymbol{\alpha} \text { Subunit (gene symbol) } & \boldsymbol{\beta} \text { Subunit (gene symbol) } \\ \alpha-3 \text { (Itga3) } & \beta-1 \text { (Itgb1) } \\ \alpha-7 \text { (Itga7) } & \\ \alpha-V \text { (Itgav) } & \end{array}$

Integrin subunits identified in utricle-derived exosomes. 
A
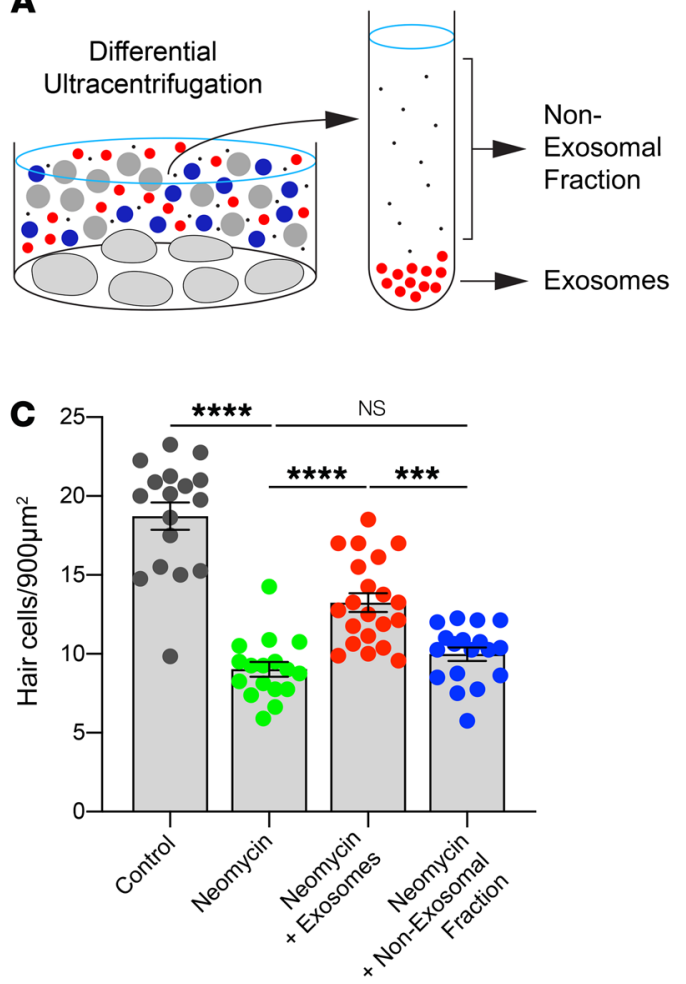

B

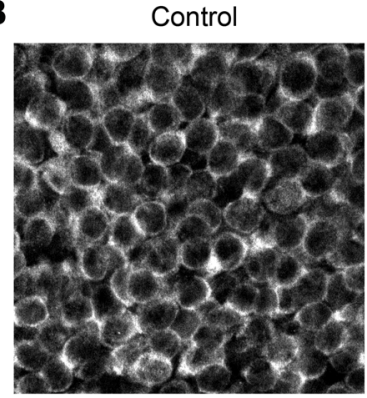

Neomycin

+ Exosomes

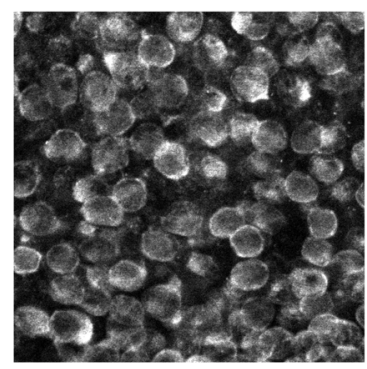

Neomycin

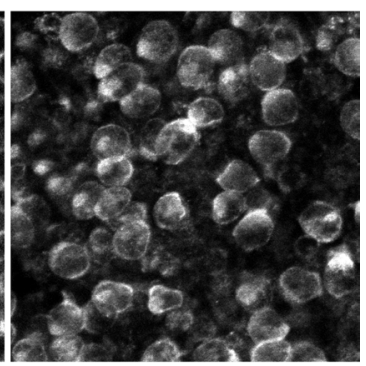

Neomycin

+ Non-Exosomal Fraction

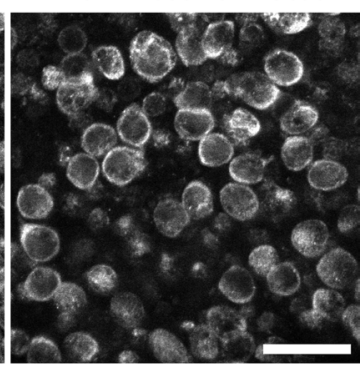

Figure 4. Isolated exosomes protect against neomycin-induced hair cell death. Utricles were cultured for 24 hours in neomycin, with or without the addition of exosomes isolated from heat-shocked utricles. (A) Exosomes and the non-exosomal fraction (supernatant) were purified from utricle-conditioned media using differential ultracentrifugation (see Figure 2B) and applied to neomycin-treated utricles. (B) Fixed utricles were labeled with the hair cell marker myosin 7a, and images were acquired using laser scanning confocal microscopy. Representative $z$ sections from surface preparations of utricle whole mounts are shown. Scale bar: $20 \mu \mathrm{m}$. (C) Neomycin caused hair cell death. Application of isolated exosomes significantly improved hair cell survival. In contrast, no protective effect was observed when the non-exosomal fraction (i.e., exosome-depleted conditioned media) was added. Each data point represents the average hair cell density of an individual utricle. $n=16-20$ utricles per condition from 4 independent experiments. Data indicate the mean \pm SEM. ${ }^{* *} P<0.001$ and ${ }^{* * *} P<0.0001$, by Brown-Forsythe and Welsh ANOVA followed by Dunnett's T3 multiple comparisons test.

A proximity ligation assay (PLA) was used to assess whether HSP7O and TLR4 interact in utricles. Two proteins were detected using primary Abs from different species, which were subsequently visualized using secondary Abs conjugated to short DNA oligonucleotides. If the 2 antigen- $\mathrm{Ab}$ complexes were in proximity (30-40 $\mathrm{nm}$ apart), the conjugated DNA oligonucleotides participated in rolling circle PCR amplification. We visualized the DNA product with labeled oligonucleotide probes to mark the sites of protein-protein interaction. The well-characterized interaction between HSP7O and its co-chaperone HSP4O was used as a positive control paradigm to validate PLA in utricles. Punctate fluorescence, indicating HSP70HSP4O interaction, was detectable in control utricles, and the number of HSP70-HSP4O puncta increased after heat shock (Figure 8, B and E). Similarly, we observed a baseline number of puncta for the interaction of HSP7O with TLR4 under control conditions, and the number of puncta increased significantly after heat shock (Figure 8, $B$ and F). Importantly, this heat shock-induced increase in HSP70TLR4 puncta was abolished in the presence of 2 different HSP70 fbAbs but not in the presence of control IgG (Figure 8, B and F), confirming the specificity of the HSP70-TLR4 interaction.

To further corroborate that HSP70 specifically interacts with TLR4 on hair cells, we used a PLA to examine the HSP70-TLR4 interaction in utricles from TLR4-cKO mice. As expected, we observed puncta indicating interaction for both HSP70-HSP4O (positive control) and HSP70-TLR4 in WT utricles (Figure 8C). Both of these interactions increased significantly after heat shock (Figure 8C). In contrast, interaction between HSP70 and TLR4 was not detected above background levels in TLR4-cKO mice under control or heat shock conditions (Figure 8, C and G). Together, these data demonstrate that exosomal HSP70 interacts with TLR4 at the hair cell surface, and the specificity of this interaction was confirmed by our data showing that either blockade of HSP7O or genetic deletion of TLR4 from hair cells abolished this interaction.

\section{Discussion}

Exosomes are mediators of the heat stress response in the inner ear. Our data reveal a biological function for exosomes in the inner ear. Previous work has shown that activation of the heat stress response improves hair cell survival in the face of toxic stress. We present evidence that exosomes are critical intercellular mediators of the protective effect of heat shock against aminoglycoside-induced hair cell death. Heat stress induced exosome release in the inner ear, consistent with data in other systems showing that heat stress affects both the quantity of exosomes released and their HSP content $(26,27,60)$. Consequently, our analyses of utricle-derived 

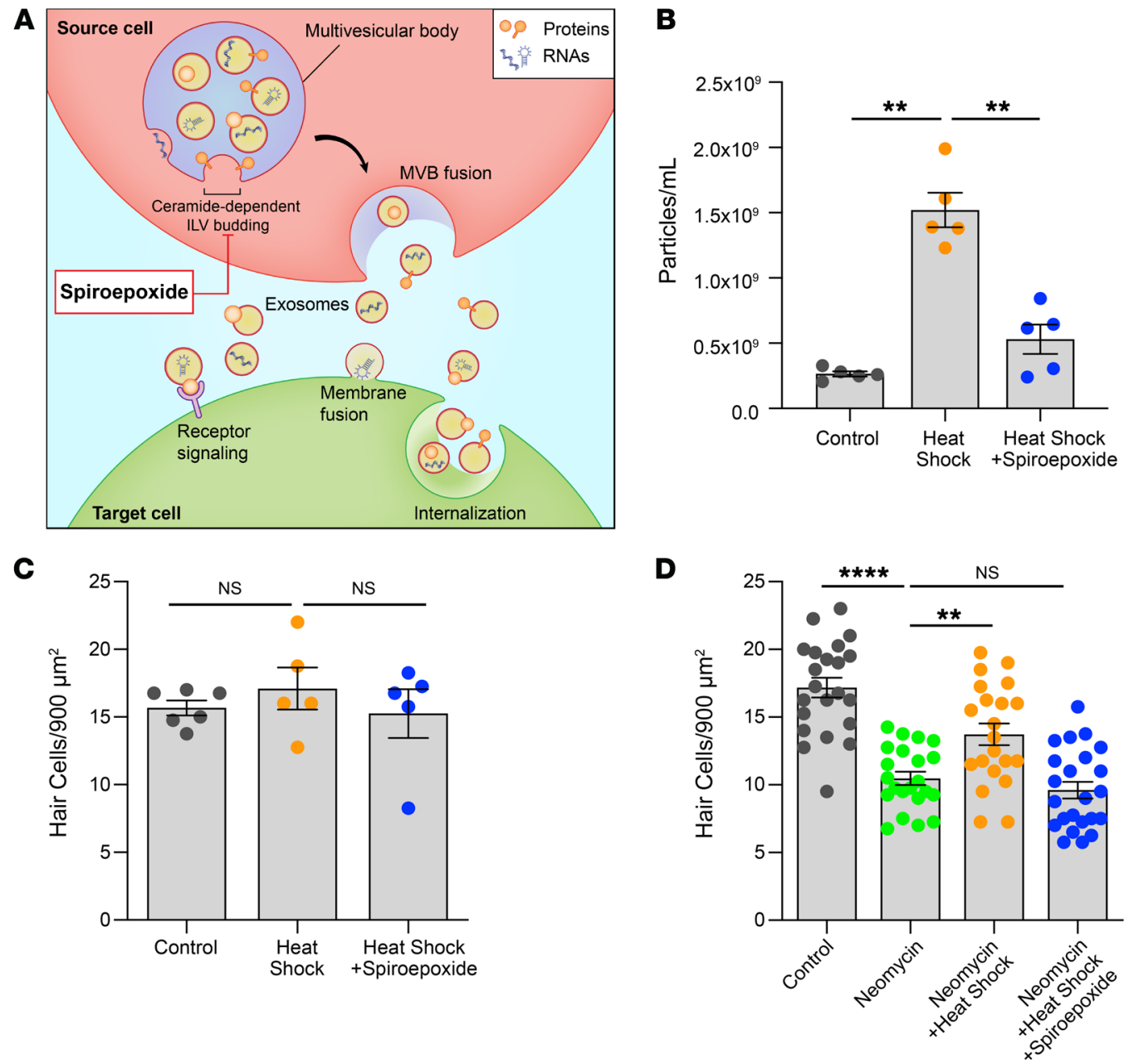

Figure 5. Exosomes are required for the protective effect of heat shock. (A) Exosomes containing proteins, nucleic acids, and lipids are released from source cells (red) and can modify the biological state of target cells (green) via a variety of interactions. Within the source cell, exosome biogenesis occurs via budding of intraluminal vesicles (ILV) into the lumen of a multivesicular body (MVB) (purple), a process that requires the sphingolipid ceramide. The N-SMase inhibitor spiroepoxide blocks ceramide production and inhibits exosome biogenesis. (B) Inhibition of exosome biogenesis reduced the number of exosome-sized particles in conditioned media from heat-shocked utricles. Data indicate the mean \pm SEM for 5 NTA captures. (C) Quantification of surviving hair cells in utricles demonstrated that reduced exosome release in the presence of spiroepoxide was not caused by cytotoxicity. $n$ = 5-6 utricles per condition. (D) Utricles were cultured for 24 hours in neomycin, with or without heat shock and with or without spiroepoxide. Neomycin caused hair cell death, whereas heat shock improved survival of neomycin-exposed hair cells. Inhibition of exosome biogenesis using spiroepoxide abolished the protective effect of heat shock. $n=21-23$ utricles (shown as individual data points) per condition. Data indicate the mean $\pm S E M$. ${ }^{* *} P<$ 0.01 and ${ }^{* * *} P<0.0001$, by Brown-Forsythe and Welsh ANOVA followed by Dunnett's T3 multiple comparisons test (B and D) or 1-way ANOVA followed by Holm-Šídák multiple comparisons test (c).

exosomes by TEM and tandem mass spectrometry revealed that they share both morphology and common protein markers with exosomes from other systems (reviewed in refs. 31, 61).

Two lines of experimentation demonstrated the critical roles of exosomes in non-cell-autonomous protection in the inner ear. First, utricle-derived exosomes protected against neomycin-induced hair cell death, whereas the non-exosomal fraction of the conditioned media was not protective. This was not due to a general increase in global protein abundance in the exosomal fraction, since both our proteomics data and our BCA analyses indicated 5- to 10-fold higher total protein content in the supernatant fraction compared with that in the exosomal fraction (data not shown). Second, inhibition of N-SMase using spiroepoxide demonstrated that exosome biogenesis is required for the protec- tive effect of heat shock. Thus, our data indicate that exosomes are both necessary and sufficient for protection against hair cell death caused by neomycin.

Inner ear exosomes carry HSP7O. Extracellular HSP70 promotes survival of hair cells exposed to ototoxic drugs; however, the mechanism by which HPS7O is secreted has been unclear (18). Our data indicate that HSP7O is released from inner ear cells by incorporation into exosomes. We detected HSP7O in isolated exosomes via tandem mass spectrometry, Western blotting, and immunoelectron microscopy. Immunoelectron microscopy showed that HSP70 appeared to be associated with the exosomal membrane. This idea was further supported by the observation that incubation of purified exosomes with a fbAb against HSP70 abolished their protective activity, suggesting that HSP70 was at least partially exposed on the exosome 


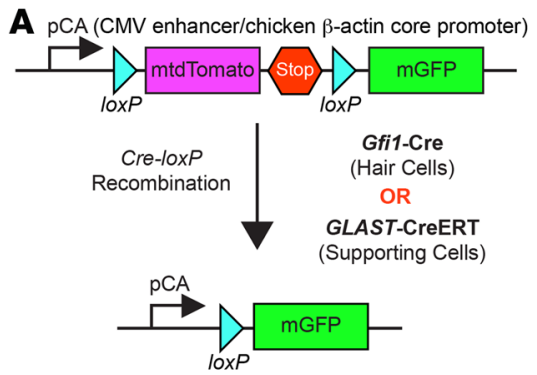

B
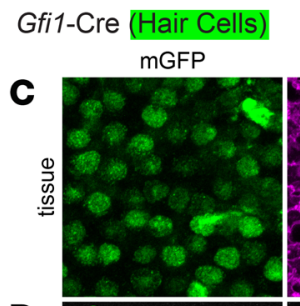

D
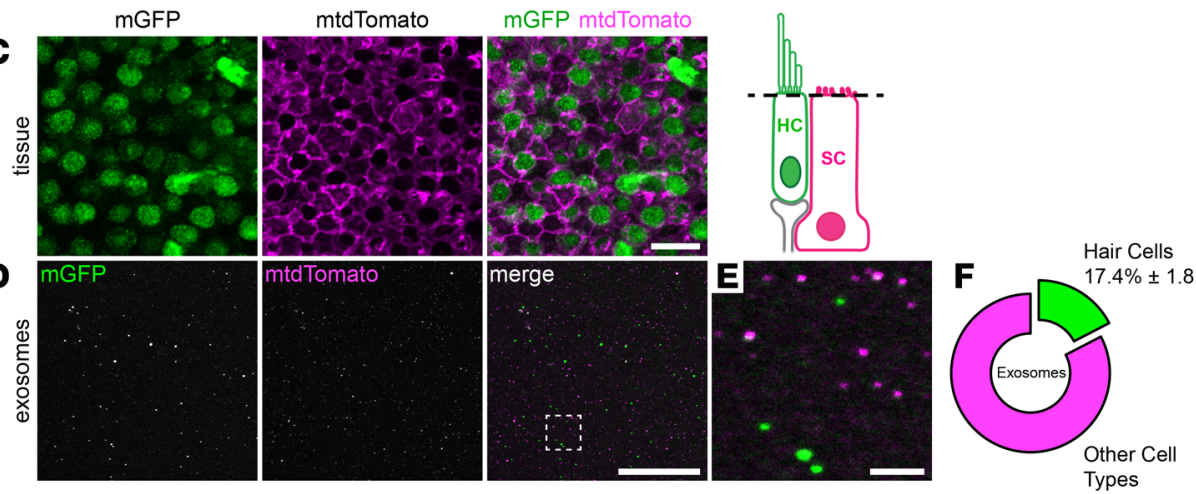

GLAST-CreER (Supporting Cells)
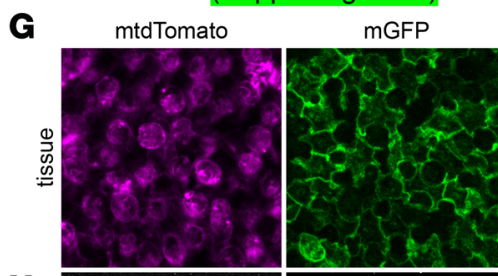

H
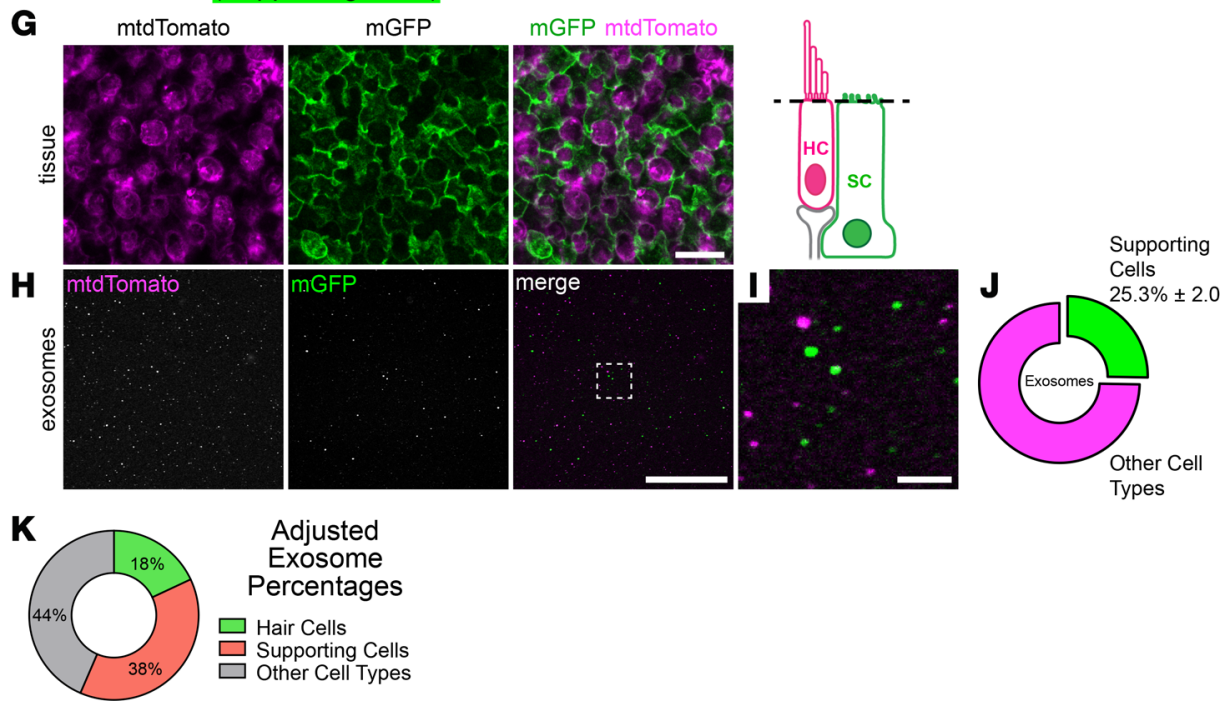

Adjusted

Exosome

Percentages

Hair Cells

Supporting Cells

Other Cell Types
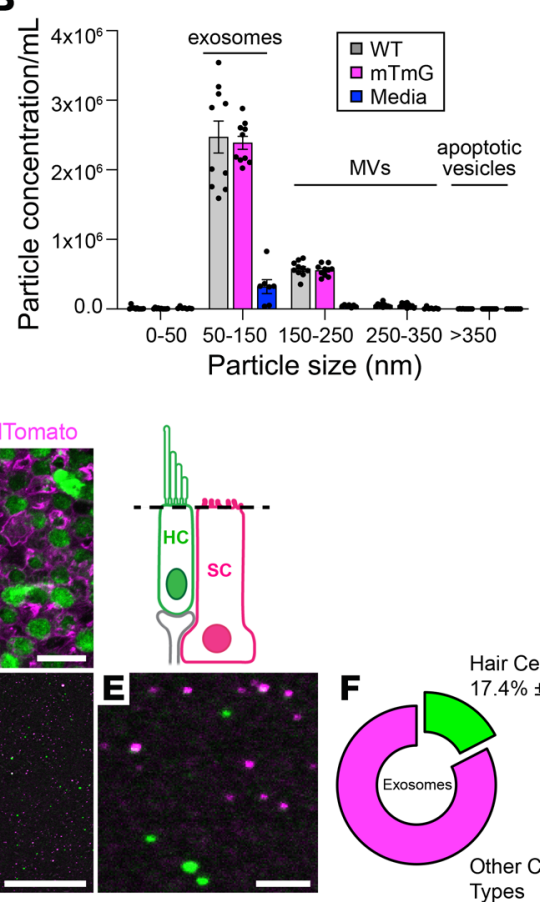

Types
Figure 6. Supporting cells release more exosomes than hair cells under heat stress. (A) mTmG double-fluorescent reporter mice constitutively express myristoylated tdTomato. When crossed with Cre recombinaseexpressing mice, the loxP-flanked mtdTomato cassette is deleted in all Cre-expressing cells, resulting in tissue-specific mGFP fluorescence. (B) NTA of conditioned media from heat-shocked utricles of mTmG mice (magenta) or age-matched WT mice (gray) shows that lipidation of fluorophores in $\mathrm{mTmG}$ mice did not affect exosome release. The culture media (blue) contributed $10 \%$ of particles to each size category. MVs, microvesicles. Data indicate the mean $\pm \mathrm{SEM}$ and are from 2 independent experiments ( $n=5$ NTA captures from 22 utricles for each condition). (C) Utricles from $\mathrm{mTmG}$ mice crossed with Cfi7-Cre mice displayed mGFP-expressing hair cells (green), whereas supporting cells retained mtdTomato expression (magenta). Schematic depicts the focal plane. (D) Fluorescence emitted from utricle-derived exosomes from mTmG mice crossed with GfiT-Cre mice. Box indicates the region magnified in $\mathbf{E}$. (F) $17.4 \%$ of utricle-derived exosomes in $\mathbf{D}$ were mGFP positive. (C) Supporting cells in $\mathrm{mTmG}$ mice crossed with CLAST-CreER mice were mGFP positive (green). All other cells retained $\mathrm{mtd}-$ Tomato expression (magenta). (H) Fluorescence emitted from utricle-derived exosomes from mTmG mice crossed with CLAST-CreER mice. Box indicates the region magnified in I. (J) $25.3 \%$ of exosomes visualized in $\mathbf{H}$ were mCFP positive. (K) Contributions of hair cells and supporting cells to the total utriclederived exosome population after taking into account Cre recombinase efficiency in hair cells (Cfit-Cre $=96.5 \%$ ) and supporting cells (CLAST-CreER $=64.5 \%)$. Results showed that $44 \%$ of exosomes were probably contributed by other cell types. Data in $\mathbf{F}$ and $\mathbf{J}$ are presented as the mean \pm SEM and are from 3 experiments ( $n=9-11$ utricles per condition). Scale bars: $10 \mu \mathrm{m}$ (C and $\mathbf{G}$ ), $50 \mu \mathrm{m}$ ( $\mathbf{D}$ and $\mathbf{H})$, and $5 \mu \mathrm{m}$ (E and $\mathbf{I})$. surface and thus accessible for Ab binding. Our results join the growing body of literature demonstrating that HSP70 is secreted via exosomes $(20,23,62,63)$. Exosomal HSP70 affects target cell protein homeostasis (64), and HSP70 on the exosome surface has repeatedly been implicated in immune cell activation, particularly in the context of cancer $(26,47-50,63)$. Notably, functional exosome-associated HSP70 appears to be important for interaction with immediate downstream receptor(s) in the inner ear, since Abs against HSP70 inhibited HSP70-TLR4 interaction in hair cells (Figure 8). This finding is in agreement with data on myeloid-derived suppressive cells, in which targeting membrane-associated HSP70 with an aptamer blocked the ability of exosomes to bind to TLR2 (65).

This work identified exosome-associated HSP70 as an indispensable factor for hair cell survival in the presence of ototoxic drugs. In addition, our proteomics analysis identified numerous other proteins associated with exosomes released from heatshocked utricles, some of which may have key roles in the pro-survival activity of inner ear exosomes.

Model for exosome-mediated protection of hair cells. Our data are consistent with a model in which utricle-derived exosomes carry surface-associated HSP70, and this exosomal HSP70 interacts with TLR4 on hair cells to promote their survival (Figure 9). Though our data suggest that a significantly greater number of exosomes were derived from supporting cells relative to hair cells, extremely limited exosome yield from mouse utricle explants precluded the sorting and analysis of subpopulations of exosomes according to their cell type of origin. Given that the isolated exosomes in this study represented the total population of exosomes released from all cells of the utricle, it is unclear whether the exosomes that promoted hair cell survival were specifically 

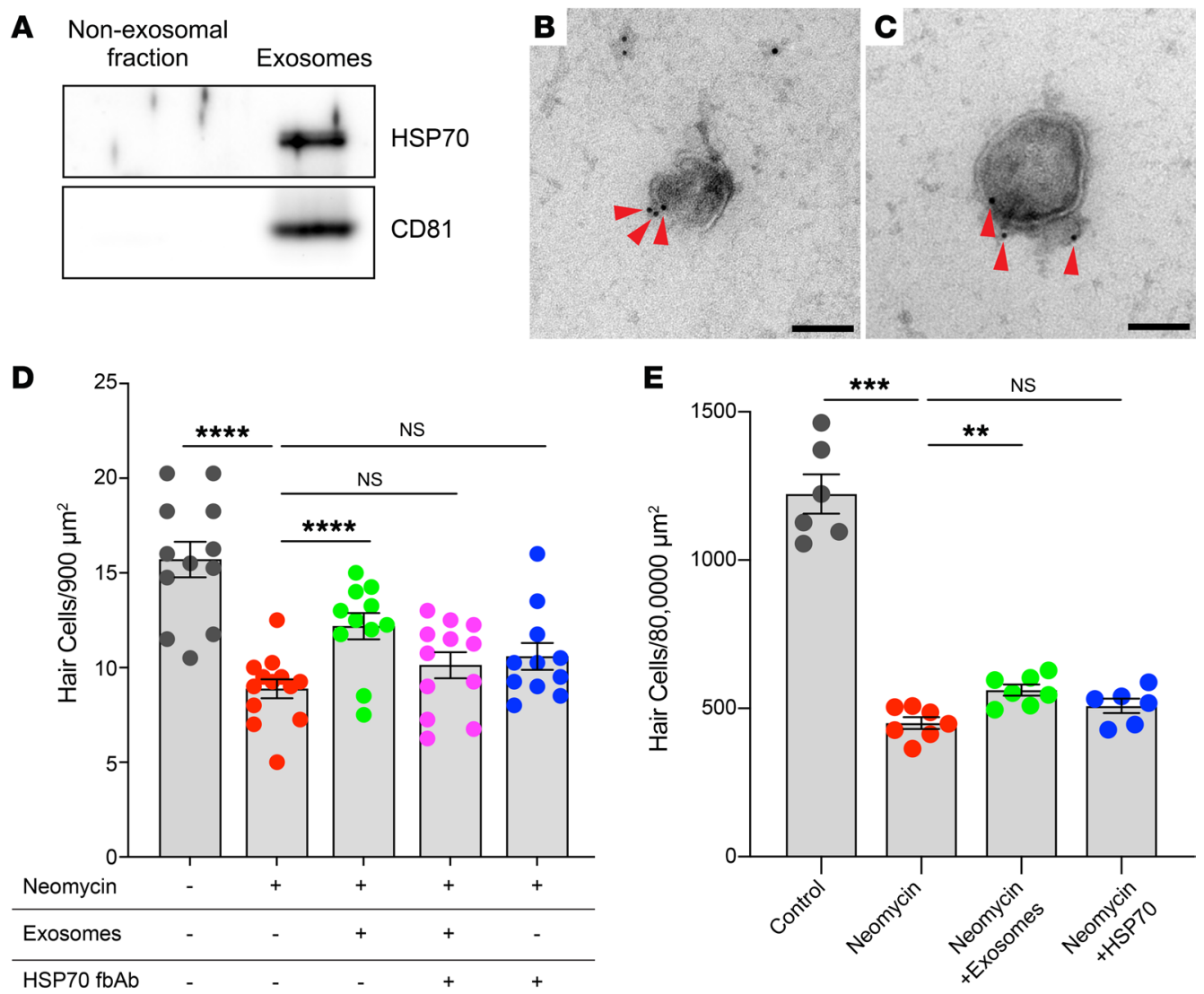

Figure 7. Exosomes contain HSP70, and exosome-associated HSP70 is required for the protective effect of exosomes. (A) Western blot shows the association of HSP70 with exosomes but not the non-exosomal fraction. Similarly, CD81 (exosome marker) was detected exclusively in exosomes. Exosomes and the non-exosomal fraction were isolated from heat-shocked utricles by differential ultracentrifugation (Figure 2B). Equivalent amounts of total protein were loaded for each lane. See complete unedited blots in the supplemental material. (B and C) Two representative Immunogold TEM micrographs of utricle-derived exosomes revealed HSP7O immunoreactivity (red arrowheads) near the exosomal membrane. Scale bars: $100 \mathrm{~nm}$. (D) HSP70 was required for the protective effect of exosomes. Utricles were cultured for 24 hours in the presence of neomycin, with or without the addition of exosomes isolated from heat-shocked utricles. Exosomes significantly protected hair cells against neomycin-induced ototoxicity. Addition of an HSP70 fbAb abolished the protective effect of exosomes. Each data point represents the average hair cell density of an individual utricle from 4 independent experiments $(n=11-13$ utricles per condition). (E) Non-exosomal HSP70 was not protective against neomycin-induced hair cell death. Utricles were cultured for 24 hours in the presence of neomycin, with the addition of either isolated exosomes or soluble (recombinant, non-exosomal) HSP7O. Exogenous HSP7O failed to protect hair cells, whereas exosomes were protective. Data indicate the mean $\pm \mathrm{SEM} .{ }^{* *} P<0.01,{ }^{* * *} P<0.001,{ }^{* * *} P<0.0001$, by Brown-Forsythe and Welsh ANOVA followed by Dunnett's T3 multiple comparisons test.

those released by supporting cells. However, our previous work (18) as well as the data presented here (Figure 1) show that heat stress induces HSP70 primarily in supporting cells, and thus it seems reasonable to posit that HSP70-positive exosomes may be released by this cell type.

Our data show that exosomes interact with TLR4 on hair cells, but the pro-survival signaling events downstream of this interaction remain unknown. The pro-survival activity of exosomal HSP7O may be independent of the chaperone function of HSP7O, as has been demonstrated in hyperoxia-induced lung injury, where extracellular HSP7O acts as a TLR4 ligand to exert a cytoprotective effect in both endothelial cells and lung tissue (59). Of interest, the protective mechanism of HSP70-containing inner ear exosomes displayed similarities to cardioprotection by plasma-derived exosomes carrying HSP7O. Here, surface-associated exosomal HSP7O interacted with TLR4 on cardiomyocytes, stimulating the downstream signaling kinases ERK1/2 and p38 MAPK to activate HSP27
(HSPB1). This exosome-driven, HSP70/TLR4-induced signaling cascade protects cardiomyocytes against ischemia/reperfusion injury $(58,66)$. A similar mechanism may underlie the protective effect of exosomes against hair cell death. Ongoing analysis of the transcriptomic changes induced in exosome-treated hair cells may illuminate the pro-survival responses within hair cells.

Exosomes as inner ear therapeutics. Death of sensory hair cells is the central event leading to permanent hearing loss caused by ototoxic drugs, noise exposure, and aging $(67,68)$. Identification of the mechanisms that promote hair cell survival will inform the rational design of therapies to prevent hearing loss. Here, we present evidence that exosomes mediate intercellular communication in the inner ear and protect against ototoxicity. Thus, exosomes may represent a means of clinical intervention to protect hair cells in vivo during cellular stress. Future experiments will determine whether exosome-mediated hair cell protection extends to protection against other ototoxic stressors. 
A

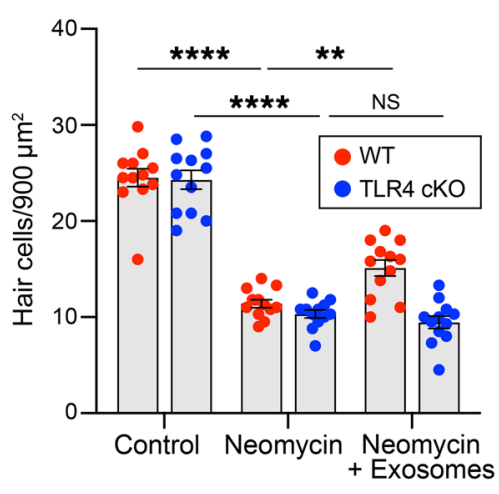

B

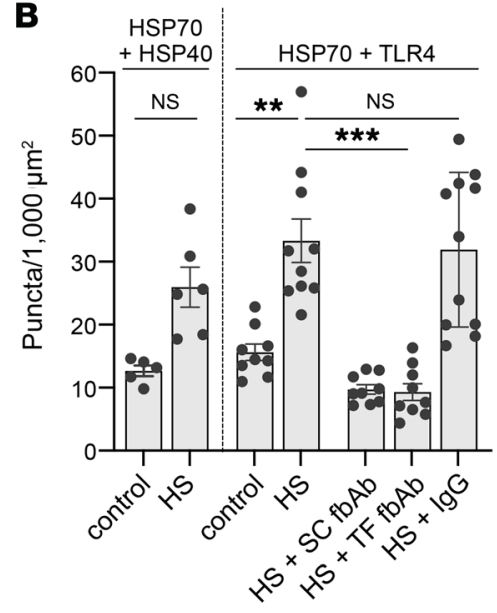

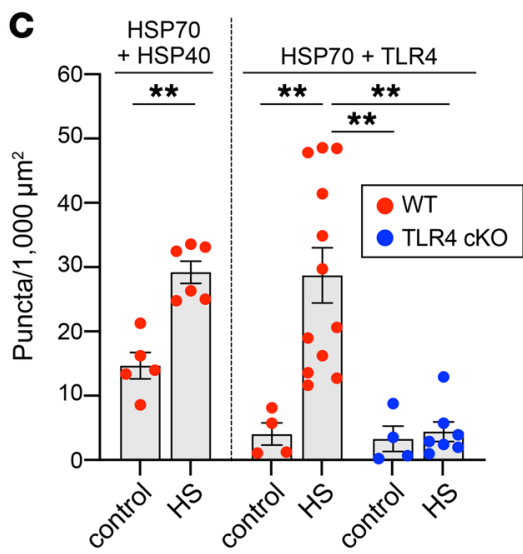
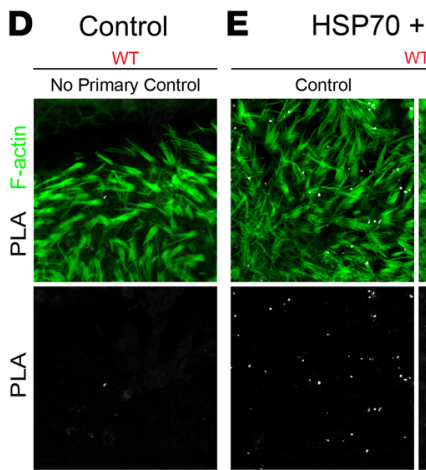

$\mathbf{F}$
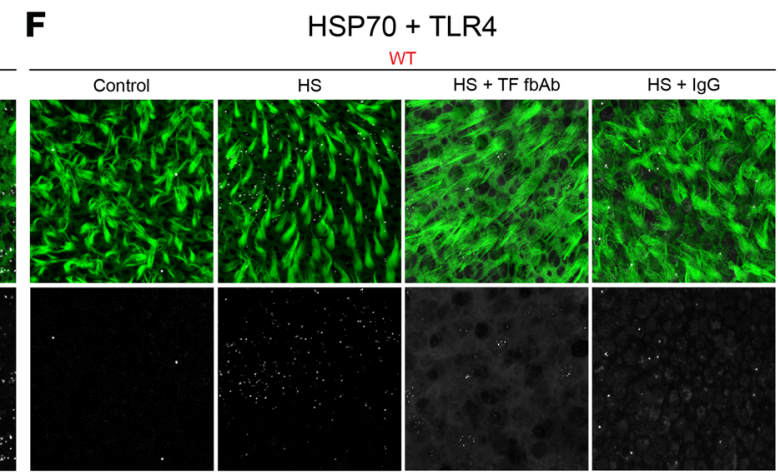

G $\quad$ HSP70 + TLR4
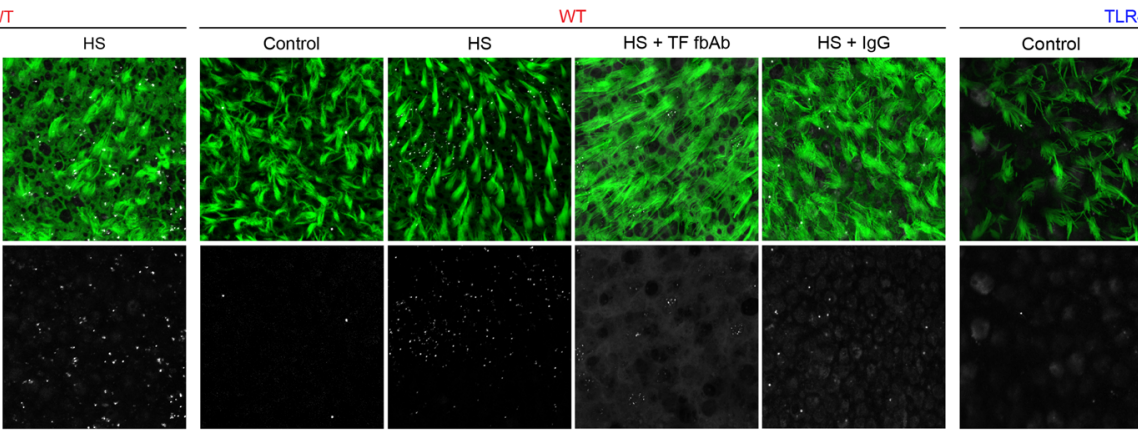

TLR4 CKO

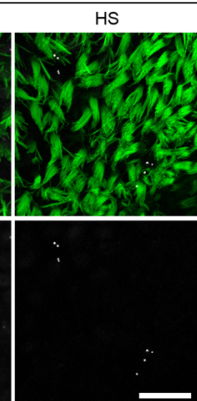

Figure 8. The protective effect of exosomes requires interaction of exosomal HSP70 with TLR4 on hair cells. (A) Exosomes improved hair cell survival in neomycin-exposed utricles from control (WT) littermates (red) but not from hair cell-specific TLR4-cKO mice (blue). Data indicate the mean \pm SEM ( $n=12$ utricles per condition). ${ }^{* *} P<0.01$ and ${ }^{* * *} P<0.0001$, by 2 -way ANOVA with Holm-Šídák multiple comparisons test. (B and C) A PLA was performed to detect interaction between exosomal HSP70 and HSP4O or HSP70 and TLR4. (B) Two different fbAbs against HSP70 (HS) abolished the interaction between HSP70 and TLR4, whereas IgG had no effect. SC, Santa Cruz Biotechnology; TF, Thermo Fisher Scientific. (C) Heat shock increased the interaction between HSP70 and HSP40 and between HSP70 and TLR4 in WT utricles. Hair cell-specific deletion of TLR4 abolished the PLA signal in heat-shocked utricles from TLR4-cKO mice. Data in B and C indicate the mean \pm SEM and are shown as the average number of puncta per $1000 \mu \mathrm{m}^{2}(n$ $=4-12$ utricles per condition). ${ }^{* *} P<0.01$ and ${ }^{* * *} P<0.001$, by Brown-Forsythe and Welsh ANOVA followed by Dunnett's T3 multiple comparisons test. (D-G) Confocal images of PLA signals in utricles from WT (D-F) or TLR4-cKO (G) mice under control or heat shock conditions. Top row, F-actin (green) and PLA signal (white); bottom row, PLA signal only (white). (D) Negative control (no primary Ab). (E) Heat shock induced HSP70 interaction with HSP40 in WT utricles. (F) Heat shock increased HSP70 interaction with TLR4 in WT utricles. HSP70 fbAbs inhibited interaction between HSP70 and TLR4 in WT utricles, independently of heat shock, whereas control IgG had no effect. (G) Hair cell-specific deletion of TLR4 abolished the HSP70-TLR4 interaction in TLR4-cKO mice. Scale bar: $20 \mu \mathrm{m}$.

We identified the protein cargo of utricle-derived exosomes using tandem mass spectrometry and demonstrated that HSP7O is a critical component of this exosomal cargo (Figure 7D and Figure 8B). These data may allow for the scalable production of hair cellprotective exosomes in vitro. Exosomes have garnered significant interest in recent years for the therapeutic delivery of proteins, nucleic acids, and small molecules (69-73), and our data indicate that they may be leveraged as nanocarriers to deliver therapeutics to treat inner ear disorders. In contrast to viral and nonviral delivery systems, exosomes have the advantages of a large packaging capacity, biocompatibility, nonimmunogenicity, and the innate ability to cross biological membranes, including the blood-brain barrier (61). Like the brain, the inner ear is separated from systemic circulation by a semipermeable, multicomponent barrier (blood-labyrinth barrier) $(74,75)$. Engineered exosomes can deliver designer cargo to the brain following systemic administration
(76). Similar results may be achievable in the inner ear using specific targeting proteins identified in our proteomics analysis. The complement of integrins on exosomes determines their tissue tropism in other systems (40). Integrin subunits, $\alpha-3, \alpha-7, \alpha-\mathrm{V}$, and $\beta-1$ were detected in our proteomics analysis, and their assembly into heterodimers may direct exosomes to specific inner ear cell types. Exosomes may also increase the delivery efficiency of locally administered inner ear therapies, as has been demonstrated for exosome-associated adeno-associated virus (AAV), which improved hair cell infectivity as compared with naked AAV (77).

Taken together, our results indicate that exosomes represent a previously undescribed mechanism of intercellular communication in the inner ear that can mediate nonautonomous survival signaling and protect sensory hair cells against lethal stresses. Exosomes may be engineered to deliver therapeutic molecules to the inner ear to prevent or reverse hearing loss. 


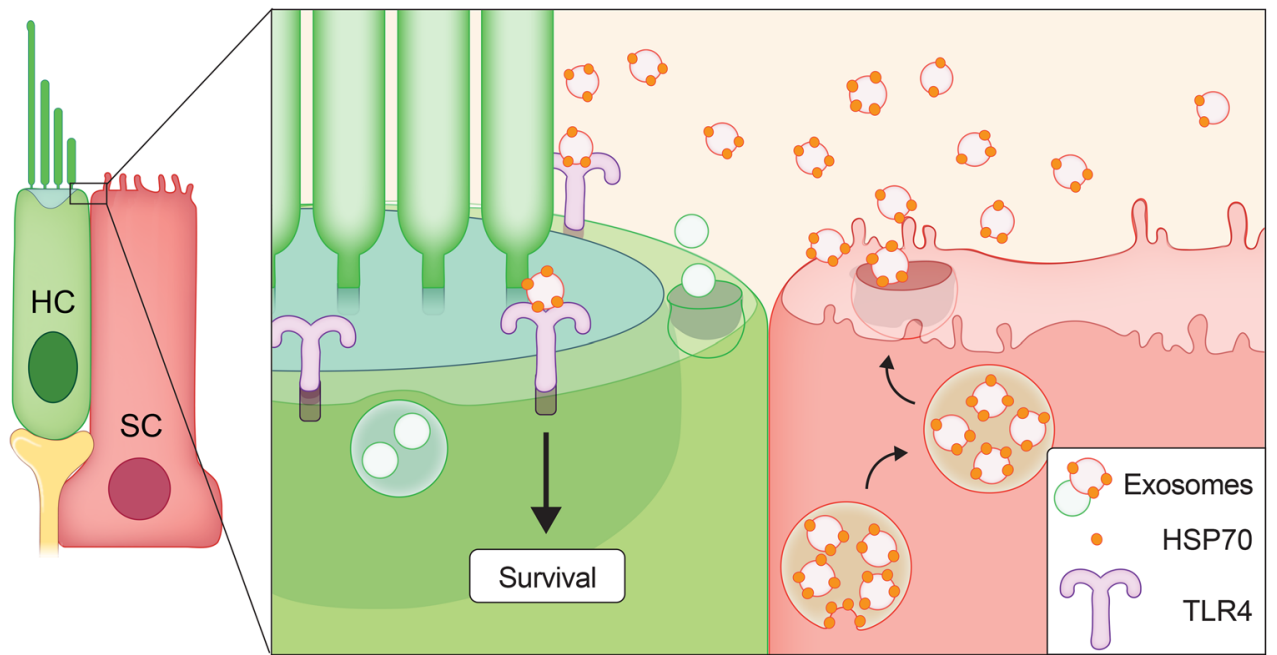

Figure 9. Model for the role of exosomes as mediators of protection against hair cell death caused by ototoxic drugs. Our data are consistent with a model in which heat stress induces HSP7O expression, predominately in supporting cells (Figure 1). Some of this HSP7O associates with exosomes (Table 1 and Figure 7, A and $B)$ that are released into the extracellular environment (Figure 2A and Figure 5B). HSP7O-carrying exosomes interact with TLR4 on the hair cell's surface (Figure 8) to stimulate a pro-survival response (Figure 4, B and C, and Figure 5D). The significance of hair cell-derived exosomes (Figure 6, C-K) remains under investigation.

\section{Methods}

Mice. Four- to 8-week-old CBA/J mice (The Jackson Laboratory, stock no. 000656) were used as controls throughout, except in experiments using TLR4-cKO mice, in which either littermates or C57BL/6J mice (as indicated) were used as controls. Approximately equal numbers of male and female mice were used in each experiment.

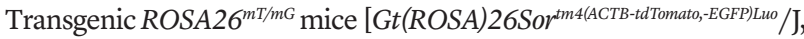
The Jackson Laboratory, stock no. 007676], referred to as $\mathrm{mTmG}$ mice, were used for visualization of EVs. These Cre-reporter mice express membrane-targeted (myristoylated) tandem dimer Tomato (mtdTomato) before Cre recombination and mGFP after Cre-mediated excision of tdTomato (44). Both fluorophores are myristoylated, promoting their ubiquitous insertion into membranes. To differentially label EVs derived from hair cells and supporting cells, 2 complementary breeding schemes were used. To label hair cells with mGFP and supporting cells with mtdTomato, hair cell-specific Gfi1 ${ }^{\mathrm{Cre} /+}$ mice (generated by Lin Gan, University of Rochester, Rochester, New York, USA, and provided by Ronna Hertzano, University of Maryland, College Park, Maryland, USA) were used. Gfi1 ${ }^{\text {Cre } /+}$ mice express Cre recombinase in the Gfil locus, which is required for hair cell differentiation and survival (78). Offspring were backcrossed with ROSA$26^{m T m G / m T m G}$ to produce $G f i 1^{C r e /+} R O S A 26^{m T m G / m T m G}$ mice. To label supporting cells with mGFP and hair cells with mtdTomato, supporting cell-specific GLAST-CreER mice [Tg(Slc1a3-Cre/ERT)1Nat, The Jackson Laboratory, stock no. 012586] were used. GLAST-CreER mice express tamoxifeninducible Cre recombinase under control of the GLAST promoter (79). GLAST-CreER ROSA26 $6^{m T m G /+}$ offspring were backcrossed with ROSA$26^{m T m G / m T m G}$ mice to obtain GLAST-CreER ROSA2 $6^{m T m G / m T m G}$ offspring. Cre recombination was induced at $\mathrm{P} 21$ by intraperitoneal injection of tamoxifen $(0.225 \mathrm{mg} / \mathrm{g}$ BW; MilliporeSigma). Cre recombination efficiency was established by crossing either Gfil-Cre mice or GLAST-CreER mice with

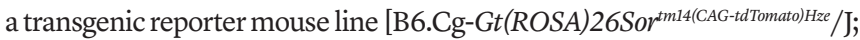
The Jackson Laboratory, stock no. 007914], followed by quantification of either tdTomato-positive hair cells (Gfi1-Cre, 96\% recombination efficiency) or tdTomato-positive supporting cells (GLAST-CreER, 65\% recombination efficiency) in utricle explants (80).

Hair cell-specific TLR4-cKO mice were generated by crossing B6.Cg-Tg(Atoh1-cre)1Bfri/J mice (The Jackson Laboratory, stock no. 011104) with B6(Cg)-Tlr $4^{\text {tml.1Karp }} / J$ mice (The Jackson Laboratory, stock no. 024872). In B6.Cg- $T$ (Atoh1-cre)1Bfri/J mice, the mouse
Atoh1 regulatory sequences direct Cre recombinase expression in all hair cells and a small percentage of supporting cells (81). B6(Cg)Tlr $4^{\text {tm1.1Karp }} / \mathrm{J}$ mice contain loxP sites flanking exon 3 of the Tlr 4 gene. Offspring that inherited the Atoh1-Cre transgene had Tlr4 conditionally deleted from hair cells and are referred to herein as TLR4-cKO mice. Offspring that did not inherit the Atoh1-Cre transgene were used as littermate controls.

Utricle culture. Explanted mouse utricles were used as an in vitro preparation of inner ear hair cells and supporting cells. Mice were euthanized by $\mathrm{CO}_{2}$ inhalation followed by decapitation. Utricles were excised and cultured as previously described $(82,83)$. Briefly, the bony labyrinth was removed and placed in Medium 199 (Gibco, Thermo Fisher Scientific) at $37^{\circ} \mathrm{C}$, and utricles were dissected under sterile conditions before transfer to $1 \mathrm{~mL}$ complete medium (DMEM/F12 with Phenol Red, Gibco, Thermo Fisher Scientific), 5\% heat-inactivated FBS (Gibco, Thermo Fisher Scientific), and $50 \mathrm{U} / \mathrm{mL}$ penicillin G (MilliporeSigma). Utricles were cultured overnight in a 24 -well tissue culture plate at $37^{\circ} \mathrm{C}$ and $5 \% \mathrm{CO}_{2}$ before any experimental manipulation.

As FBS contains large numbers of exosomes (84), experiments in which vesicles were to be isolated and/or analyzed were performed in serum-free culture medium (DMEM/F12, $50 \mathrm{U} / \mathrm{mL}$ penicillin $\mathrm{G}$ ). Utricles initially cultured in serum-containing media were rinsed and transferred to serum-free medium. Imaging of fluorescently labeled exosomes was performed in serum-free medium without phenol red.

Heat shock. Utricles and their culture medium $(1 \mathrm{~mL})$ were transferred to sterile microcentrifuge tubes and placed into a $43^{\circ} \mathrm{C}$ water bath for 30 minutes. Control (no heat shock) utricles were transferred into microcentrifuge tubes and placed in the incubator at $37^{\circ} \mathrm{C}$ for 30 minutes. Following incubation, all utricles were transferred to complete medium in a tissue culture plate and returned to the incubator under standard culture conditions for 2 hours.

NTA. NTA was performed using a NanoSight NS300 controlled by NTA software, version 3.1 (Malvern Panalytical), to measure the concentration and size distribution of EVs. Samples were pushed through a fluidics flow chamber at a constant flow rate using a syringe pump (Harvard Apparatus). The scattered light from vesicles illuminated with a $488-\mathrm{nm}$ laser was recorded at 30 frames per second using a sCMOS camera 3 times for 60 seconds (Figure 2A and Figure $5 \mathrm{~B}$ ) or 5 times for 30 seconds (Figure 6B). The camera sensitivity for each cap- 
ture was kept identical within the same experiment (15 in Figure 2A and Figure 5B and 13 in Figure 6B). The detection threshold for particle analysis was set at 3 .

Exosome purification. Samples were of 2 categories: (a) minimally processed conditioned media or (b) purified and concentrated exosomes. Minimally processed conditioned media were analyzed to characterize the total population of EVs released from utricles (Figure 2A and Figure $6 \mathrm{~B})$. To remove large particles and cellular debris before NTA, conditioned medium was processed either by centrifugation $(2000 \times \mathrm{g}$ for 10 minutes; Figure $2 \mathrm{~A})$ or filtration $(0.2-\mu \mathrm{m}$ syringe filter; Figure $6 \mathrm{~B})$.

Exosomes were purified from conditioned medium either by ultracentrifugation or size-exclusion chromatography (SEC). Ultracentrifugation provided a higher yield and was used for most experiments. SEC was performed to generate high-purity samples for proteomics analysis (Figure 3 and Tables 1 and 2).

Ultracentrifugation method. Exosomes were isolated from utricle-conditioned medium using an abbreviated version of a previously described protocol (29). Cells and large cellular debris were removed by centrifugation $\left(300 \times g, 10\right.$ minutes, $\left.4^{\circ} \mathrm{C}\right)$, followed by sedimentation of large vesicles and additional cellular debris $(10,000 \times g, 30$ minutes, $4^{\circ} \mathrm{C}$ ). Exosomes were pelleted by subjecting the supernatant from the second spin to a high-speed centrifugation step $(100,000 \times g$, 70 minutes, $4^{\circ} \mathrm{C}$ ) in polycarbonate tubes (349622, Beckman Coulter) using a TLA-100.3 rotor and an Optima MAX-XP ultracentrifuge (Beckman Coulter). The exosome pellet was resuspended in PBS or culture medium by trituration with a micropipette.

Exosomes for proteomics analysis were purified by ultrafiltration and SEC. Cellular debris and large vesicles were removed from conditioned medium by filtration through a 0.22- $\mu$ m PVDF syringe filter (Millex Durapore, MilliporeSigma). Vesicles were concentrated using a centrifugal concentrator (5000 MWCO, Sartorius VS15T11 Vivaspin Turbo 15) at $3000 \times g$. The concentrated sample $(250-500 \mu \mathrm{L})$ was separated on a Superose 6 Increase 10/300 GL SEC column (equilibrated with PBS, pH 7.4) using an ÄKTA Pure 25 chromatography system (both from GE Healthcare Life Sciences) at $4^{\circ} \mathrm{C}$. A constant flow rate of $0.5 \mathrm{~mL} / \mathrm{min}$ was maintained throughout, and UV absorbance $(280 \mathrm{~nm}$ ) was continuously recorded. Eluted sample fractions were collected every $1 \mathrm{~mL}$ for column volumes of 0 to 1 and every $2 \mathrm{~mL}$ for column volumes of 1 to 1.5. Exosome-containing fractions were identified according to the UV absorption and by NTA of each fraction. The exosomal and non-exosomal fractions (containing secreted proteins and protein complexes) were separately pooled and concentrated using centrifugal concentrators (3000 MWCO, Sartorius VS15T91 Vivaspin Turbo 15) before proteomics analysis.

TEM and immunoelectron microscopy. TEM was used to visualize purified utricle exosomes. Fixation and embedment were performed as previously described (29). Briefly, isolated exosomes were resuspended in $2 \%$ paraformaldehyde (PFA), deposited as droplets onto Formvar/carbon-coated grids, and rinsed in PBS before fixation in 1\% glutaraldehyde. Samples were rinsed in double-distilled $\mathrm{H}_{2} \mathrm{O}\left(\mathrm{dd}_{2} \mathrm{O}\right)$, contrasted in uranyl-oxalate solution ( $\mathrm{pH} 7$ ), and embedded in $2 \%$ methyl cellulose and 4\% uranyl acetate at a ratio of 9:1. Excess solution was blotted on filter paper, and grids were air dried.

Immunoelectron microscopy was used to visualize the association of HSP7O with utricle-derived exosomes. Exosomes were fixed and deposited on Formvar/carbon-coated grids. Grids were rinsed twice by placing them onto a $100-\mu \mathrm{L}$ drop of PBS, followed by 2 washes in $50 \mathrm{mM}$ glycine in PBS. Samples were blocked in 5\% BSA (A3294, MilliporeSigma) in PBS for 10 minutes before incubation with an HSP70 Ab (sc-33575, Santa Cruz Biotechnology) and then diluted 1:5 in blocking solution for 2 hours. Control samples were incubated in blocking solution alone. Samples were rinsed 3 times in wash buffer (0.1\% BSA in PBS). Secondary staining was performed for 30 minutes using $10 \mathrm{~nm}$ gold-conjugated goat anti-rabbit $\operatorname{IgG}$ $\mathrm{F}\left(\mathrm{ab}^{\prime}\right) 2(\mathrm{H}+\mathrm{L}) \mathrm{Ab}$ (15731, BBI Solutions/Ted Pella), diluted 1:40 in blocking solution. Samples were rinsed 4 times in PBS, fixed in $1 \%$ glutaraldehyde in PBS, and then rinsed 4 times in $\mathrm{ddH}_{2} \mathrm{O}$. Samples were contrasted and embedded as described above.

Images were collected using a JEM-2100 transmission electron microscope (JEOL) equipped with an Orius SC1000B camera (Gatan) and DigitalMicrograph software. Post-acquisition processing was performed using ImageJ2 software (NIH) (85).

Tandem mass spectrometry. Utricles $(n=160)$ were dissected and cultured in DMEM/F12 medium supplemented with $50 \mathrm{U} / \mathrm{mL}$ penicillin G and G-5 serum-free supplement (Thermo Fisher Scientific) as a chemically defined substitute for FBS. Utricles were heat shocked, and exosomes were purified from conditioned media via the SEC-based method described above. Samples were flash-frozen in liquid nitrogen and stored at $-80^{\circ} \mathrm{C}$. Samples were essentially processed and analyzed as in normal high-sensitivity, bottom-up proteomics using sodium dodecanoate (86) with postextraction clean-up using STop And Go Extraction (STAGE) tips (87).

Raw data were abstracted to the mgf format using MSConvert from the ProteoWizard 3.0.6839 package. These files were entered into the Mascot search engine against the MaxQuant common contaminants database (Max Planck Institute of Biochemistry) and the SWISS-PROT protein sequence databases for Homo sapiens, Mus musculus, and Pichia pastoris (02-27-2018 release). GO term enrichment analyses were performed in PANTHER, version $13.1(35,36)$. Redundancy within lists of enriched GO terms was minimized using REViGO (37), with similarity set to 0.5 .

Modeling ototoxicity in vitro. Utricles were treated with neomycin to induce hair cell death. Neomycin sulfate solution $(140 \mathrm{mg} / \mathrm{mL}$, VetOne) was added to culture medium to a final concentration of 2.5 $\mathrm{mM}$. Neomycin-containing culture medium was equilibrated in the incubator $\left(37^{\circ} \mathrm{C}, 5 \% \mathrm{CO}_{2}\right)$ for at least 2 hours before use.

Application of exogenous exosomes. Twenty to 24 utricles were cultured, heat shocked, and transferred to serum-free culture medium. Conditioned medium was collected 24 hours later and processed via differential ultracentrifugation as described above, resulting in a pellet containing exosomes and a supernatant containing extracellular macromolecules not associated with exosomes (non-exosomal fraction). The exosome pellet was resuspended in serum-free, neomycin-containing medium to create neomycin plus exosomes culture medium. Neomycin was added to the non-exosomal fraction to create neomycin plus non-exosomal fraction culture medium. Culture media were allowed to equilibrate for 2 hours in the incubator. Following equilibration, freshly dissected utricles were transferred to each culture medium, incubated for 24 hours, and then fixed and processed as detailed below.

Pharmacologic inhibition of exosome biogenesis. Utricles were treated with spiroepoxide (282108-77-4, Santa Cruz Biotechnology), an inhibitor of N-SMase, to inhibit exosome biogenesis. N-SMase cat- 
alyzes production of ceramide, which is necessary for exosome production in some systems (41). Spiroepoxide was dissolved in DMSO and added to culture medium at a final concentration of $0.375 \mu \mathrm{M}$. Simultaneous exposure to neomycin and spiroepoxide was performed by addition of both drugs to the culture medium, followed by equilibration of the media for 2 hours, after which utricles were incubated with the media for 24 hours. Control utricles were exposed to DMSO (vehicle control). After 24 hours, utricles were fixed and processed as detailed below.

Immunohistochemistry and confocal microscopy. Utricles were fixed in 4\% PFA in PBS for 30 minutes at room temperature and then incubated for 2 hours at room temperature in blocking solution $(0.5 \%$ Triton X-100, 2\% BSA, and 0.8\% normal goat serum [NGS] in PBS). Hair cells were labeled overnight at $4^{\circ} \mathrm{C}$ with mouse anti-myosin VIIa Ab (1:100, MYO7A 138-1, Developmental Studies Hybridoma Bank), followed by Alexa Fluor 546-conjugated goat anti-mouse IgG (1:500, Invitrogen, Thermo Fisher Scientific) for 4 hours at room temperature. F-actin was labeled with Alexa Fluor 647 Phalloidin (1:60, Molecular Probes) for 45 minutes at room temperature, and nuclei were stained with Hoechst 33342 (1:500, Molecular Probes) for 5 minutes. All incubation steps were performed in blocking solution.

Labeled utricles were mounted in Fluoromount-G (Southern Biotech) and imaged using a $\times 40$ 1.4 NA Plan-Apochromat oil objective and an Axiovert 200M inverted microscope with a confocal scan head (LSM780) equipped with a GaAsP detector and controlled by Zen software (all from Zeiss). All samples were imaged under identical acquisition settings. Surviving hair cells within the extrastriolar region of each utricle were counted manually within 5 regions of interest (ROI) and averaged to reflect the mean hair cell density per utricle. For consistency throughout the manuscript, hair cell density was normalized to the average hair cell count per 900$\mu \mathrm{m}^{2}$ utricle area (except in Figure 7E).

Quantification of labeled exosomes. Utricles from $\mathrm{Gfi}^{\mathrm{Cre} /+} \mathrm{ROSA}$ $26^{m T m G /+}$ and tamoxifen-treated GLAST-CreER ROSA26 $6^{m T m G / m T m G}$ mice (described above) had differentially labeled hair cells and supporting cells. The membrane-associated reporter fluorophores incorporate into exosomes and report the cell type of origin of a particular exosome. The relative abundance of fluorescent hair celland supporting cell-derived EVs in conditioned medium from heatshocked $\mathrm{Ffi1}^{\mathrm{Cr} / \mathrm{+}}$ ROSA $26^{\mathrm{mTmG/+}}$ utricles was determined using laser scanning confocal microscopy.

Utricles ( $n=9-11$ per biological replicate) were dissected, heat shocked, rinsed, and transferred to $40 \mu \mathrm{L}$ of phenol red-free, serumfree medium on a 96-well tissue culture plate. Conditioned medium was collected 24 hours later, and cellular debris was removed by centrifugation (10,000 $\times g, 10$ minutes). The exosome-containing supernatant was imaged using a $\times 631.4$ NA Plan Apochromat oil objective and an Axiovert 200M inverted microscope with a confocal scan head (LSM780) controlled by Zen software (all from Zeiss) as previously described $(45,46)$. Briefly, a $10-\mu \mathrm{L}$ droplet was placed on a 35-mm glass coverslip-bottomed culture dish (MatTek) and covered. Two sequential images of exosomes adherent to the coverslip were captured, with the mtdTomato signal imaged first and the mGFP second. Image acquisition was reversed for the next 2 images to account for potential photobleaching. For each sample, a second $10-\mu \mathrm{L}$ exosome droplet was imaged using the same parameters, resulting in 8 images per sample.
Using ImageJ2, images were split into separate channels and smoothed, and the threshold was applied manually. Thresholding settings were kept constant for all images in an experiment. Exosomes in each channel were automatically quantified using the "analyze particles" function, with the minimum particle area set to 2 pixels ${ }^{2}$ and no circularity restrictions (0.00-1.00). The resulting exosome counts in each channel were averaged across the 8 images collected for each sample. The experiment was repeated 3 times.

Western blot analyses. Twenty-two utricles were cultured, heat shocked, and transferred into serum-free culture medium. Conditioned culture medium was collected 24 hours later and processed via differential ultracentrifugation (see Exosome purification), resulting in a pellet containing the exosomes, and supernatant containing extracellular proteins not associated with exosomes. The exosome-depleted supernatant was further concentrated with a centrifugal filter unit (3000 MWCO; MilliporeSigma) at $4000 \times g$ and $4^{\circ} \mathrm{C}$. Both the concentrated non-exosomal supernatant and the purified exosomes were processed as follows: samples were resuspended in 1× RIPA lysis buffer (MilliporeSigma) supplemented with cOmplete Mini EDTA-Free Protease Inhibitor Cocktail (Roche), vortexed for 1 minute, and denatured at $95^{\circ} \mathrm{C}$ for 5 minutes in $1 \times$ Laemmli buffer (Bio-Rad). Protein concentration was analyzed with a BCA protein assay kit (Thermo Fisher Scientific), using half of each sample. The remaining sample was denatured at $95^{\circ} \mathrm{C}$ for 5 minutes in $1 \times$ Laemmli buffer and subjected to SDS-PAGE using NuPAGE $4 \%$ to $12 \%$ Bis-Tris gels followed by protein transfer to a $0.45-\mu \mathrm{m}$ pore PVDF membrane (both from Thermo Fisher Scientific). Membranes were blocked in $1 \%$ BSA in TBS with $0.1 \%$ Tween-20 (TBS-T). Washes were performed with TBS-T. All Abs were diluted in 1\% BSA in TBS-T. The primary Abs used were HSPA1 (PA5-28003, 1:500, Thermo Fisher Scientific) and CD81 (D5O2Q, 1:500, Cell Signaling Technology). HRP-linked secondary Ab: Anti-rabbit IgG (7074S, 1:10,000, Cell Signaling Technology). Membranes were incubated with $2 \%$ NaN3 between primary Abs to inactivate HRP (88). Protein bands were visualized by chemiluminescence using SuperSignal West Femto Duration Substrate (Thermo Fisher Scientific) and a ChemiDoc MP Imaging System (Bio-Rad).

HSP7O function-blocking Ab. Utricles $(n=40-44)$ were cultured and processed as described above (see Western blot analyses) to generate a pellet containing the exosomes and supernatant containing extracellular macromolecules not associated with exosomes. The pelleted exosome fraction was resuspended in serum-free, neomycin-containing medium to create culture medium containing neomycin plus exosomes. This medium was equilibrated for 2 hours in the incubator and divided into two 1-mL aliquots. Rabbit anti-HSP70/ anti-HSC70 (sc-33575, H-300, Santa Cruz Biotechnology) was added at a concentration of $1 \mu \mathrm{g} / \mathrm{mL}$ to 1 aliquot to create culture medium containing neomycin plus exosomes plus HSP70 fbAb. Control medium was prepared by adding anti-HSP70/HCS7O Ab at the same concentration to equilibrated serum-free, neomycin-containing culture medium (neomycin plus HSP70 fbAb). Naive utricles were transferred to each experimental culture medium and incubated for 24 hours, following by fixation, staining, and imaging as described above (see Immunohistochemistry and confocal microscopy).

Application of exogenous HSP7O. Utricles $(n=22)$ were cultured and processed as above (see Western blot analyses) to generate a pellet containing the exosomes. The pelleted exosomes were resuspend- 
ed in serum-free, neomycin-containing culture medium to create culture medium containing neomycin plus exosomes. Recombinant human HSP70 (5 $\mu$ g; ADI-ESP-555, Enzo Life Sciences) was resuspended in serum-free, neomycin-containing culture medium to create culture medium containing neomycin plus HSP70. Culture media were equilibrated for 2 hours at $37^{\circ} \mathrm{C}$. Naive utricles were transferred to each experimental culture medium and incubated for 24 hours, after which they were fixed, stained, and imaged as detailed above (see Immunohistochemistry and confocal microscopy). Quantification was performed by counting surviving hair cells in the entire posterior region of the treated utricles, followed by normalization of hair cell counts to the utricle area.

PLA. A Duolink PLA (DUO92014, MilliporeSigma) was used to visualize interaction between HSP70 and TLR4. Utricles from TLR4-cKO or WT control mice were heat shocked. Six hours later, utricles were fixed in 3\% PFA for 30 minutes, rinsed in PBS, permeabilized with $0.1 \%$ Triton $\mathrm{X}-100$ in PBS for 10 minutes, rinsed in PBS, and blocked (2\% BSA plus 10\% NGS in PBS) for 3 hours at room temperature. Utricles were incubated overnight at $4^{\circ} \mathrm{C}$ with mouse antiHSP70 Ab (C92F3A-5, Enzo Life Sciences) and rabbit anti-HSP4O Ab (ADI-SPA-400-F, Enzo Life Sciences) (control) or with mouse antiHSP70 Ab (C92F3A-5, Enzo Life Sciences) and rabbit anti-TLR4 Ab (MAB27591, R\&D Systems). HSP70 was blocked with either rabbit HSPA1A Ab (PA5-28003, Thermo Fisher Scientific) or rabbit-HSP70 $\mathrm{Ab}$ (sc33575, H-300, Santa Cruz Biotechnology) using rabbit IgG isotype (31325, Thermo Fisher) as a control. Abs were used at a final concentration of $1 \mu \mathrm{g} / \mathrm{mL}$. One utricle per well was transferred onto Nunc MiniTrays (136528, Thermo Fisher Scientific) and treated according to the manufacturer's instructions. Briefly, utricles were incubated in secondary solution $(15 \mu \mathrm{L})$ containing anti-rabbit PLUS and anti-mouse MINUS probes for 1 hour at $37^{\circ} \mathrm{C}$. Utricles were rinsed and incubated in ligation solution $(15 \mu \mathrm{L})$ for 30 minutes at $37^{\circ} \mathrm{C}$ and then rinsed and incubated in amplification solution (15 $\mu \mathrm{L}$ ) for 100 minutes at $37^{\circ} \mathrm{C}$. After a final wash, F-actin was labeled with Alexa Fluor 488 phalloidin (1:50), and nuclei were stained with Hoechst 33342 (1:15,000, both from Molecular Probes) in PBS for 30 minutes at room temperature. Utricles were imaged using either a $\times 60$ lambda 1.4 NA Plan-Apochromat oil objective or a $\times 60$ TIRF 1.49 NA Plan Apochromat oil objective and an Eclipse Ti-E inverted microscope with a hybrid confocal scan head (resonant and galvano; A1R HD) equipped with a GaAsP detection unit controlled by NISElements C software (all from Nikon); or a ×631.4 NA DIC Plan Apochromat oil objective and an Axiovert 200M inverted microscope with a confocal scan head (LSM780) equipped with an Airyscan detection unit controlled by Zen software (all from Zeiss). Identical settings for pinhole, gain, and offset were used between samples on each microscope. Acquired Airyscan data sets were processed using
Zen Desk software (Zeiss). All data sets were processed for PLA puncta quantification in ImageJ as follows: image channels were separated, and a maximum $z$ projection of the $z$ sections containing the PLA signal was performed. The threshold was applied manually, and PLA puncta were automatically quantified using the "analyze particles" function, with the minimum particle area set from 0.2 to $1 \mu \mathrm{m}^{2}$, with no circularity restrictions (0.00-1.00). Puncta were counted for 2 to 3 ROIs per utricle, averaged across the utricle, and normalized to a $1000-\mu \mathrm{m}^{2}$ area.

Statistics. All statistical analyses were performed using GraphPad Prism 8 software (GraphPad Software). The Shapiro-Wilks test was used to analyze data sets for normality. For multiple comparisons, statistical significance was determined by 1-way ANOVA followed by a Holm-Šídák multiple comparisons test (89) for data with equal variances or otherwise by Brown-Forsythe and Welsh ANOVA followed by Dunnett's T3 multiple comparisons test. Comparisons of multiple groups with independent variables was performed by 2-way ANOVA followed by a Holm-Šídák multiple comparisons test. Data are presented as the mean \pm SEM.

Study approval. All animal procedures were approved by the IACUCs of the NIDCD and the National Institute of Neurological Disorders and Stroke (NINDS) (protocol no. 1327).

\section{Author contributions}

AMB, LAM, MJAW, and LLC conceived the project. AMB, LAM, $\mathrm{MB}, \mathrm{NCW}, \mathrm{SPF}$, and LLC planned the experiments. AMB, LAM, NJAW, SPF, TQC, LW, DEA, RSP, YXW, and MB performed the experiments and interpreted the data. AMB, MB, and LLC wrote the manuscript with input from all authors. TBF, MJAW, and LLC supervised the project. The assignment of the authorship order for the first 3 authors on this manuscript is essentially chronological: AMB started the project and worked in close collaboration with LAM, who designed and performed several of the critical experiments. Together, they produced the first submitted version of the manuscript. After AMB and LAM left the laboratory, MB completed additional important experiments, performed extensive new analyses, and rewrote the manuscript.

\section{Acknowledgments}

We are grateful to Imre Mäger and Samir El Andaloussi for guidance regarding exosome purification and analysis techniques. We thank Erina He for the illustrations in Figure 1D, Figure 5A, Figure 6, C and G, and Figure 9. We thank Matthew Kelley and Wade Chien for helpful comments on the manuscript. This research was supported by the Division of Intramural Research of the NIDCD (1ZIADC000079, to LLC, 1ZICDC000081, to RSP, and DC000039, to TBF).
1. World Health Organization. WHO Global Estimates on Prevalence of Hearing Loss. http://www. who.int/deafness/estimates/en. Accessed March 6, 2020.

2. Francis SP, Kramarenko II, Brandon CS, Lee FS, Baker TG, Cunningham LL. Celastrol inhibits aminoglycoside-induced ototoxicity via heat shock protein 32. Cell Death Dis. 2011;2:e195.

3. Francis SP, et al. A novel role of cytosolic protein synthesis inhibition in aminoglycoside ototoxici- ty. J Neurosci. 2013;33(7):3079-3093.

4. Hailey DW, Esterberg R, Linbo TH, Rubel EW, Raible DW. Fluorescent aminoglycosides reveal intracellular trafficking routes in mechanosensory hair cells. JClin Invest. 2017;127(2):472-486.

5. Huth ME, Ricci AJ, Cheng AG. Mechanisms of aminoglycoside ototoxicity and targets of hair cell protection. Int JOtolaryngol. 2011;2011:937861.

6. Marcotti W, van Netten SM, Kros CJ. The amino- glycoside antibiotic dihydrostreptomycin rapidly enters mouse outer hair cells through the mechano-electrical transducer channels. J Physiol (Lond). 2005;567(Pt 2):505-521.

7. Warchol ME. Cellular mechanisms of aminoglycoside ototoxicity. Curr Opin Otolaryngol Head Neck Surg. 2010;18(5):454-458.

8. Yoshida N, Kristiansen A, Liberman MC. Heat stress and protection from permanent acoustic injury in mice. J Neurosci.1999;19(22):10116-10124. 
9. Cunningham LL, Brandon CS. Heat shock inhibits both aminoglycoside- and cisplatin-induced sensory hair cell death. JAssoc Res Otolaryngol. 2006;7(3):299-307.

10. Taleb M, Brandon CS, Lee FS, Lomax MI, Dillmann WH, Cunningham LL. Hsp70 inhibits aminoglycoside-induced hair cell death and is necessary for the protective effect of heat shock. JAssoc Res Otolaryngol. 2008;9(3):277-289.

11. Baker TG, et al. Heat shock protein-mediated protection against cisplatin-induced hair cell death. JAssoc Res Otolaryngol. 2015;16(1):67-80.

12. Hawkins JE. Comparative otopathology: aging, noise, and ototoxic drugs. In: Hawkins JE, Lawrence M, Work WP, eds. Otophysiology. Basel, Switzerland: Karger Publishers; 1973:125-141.

13. Bohne BA, Rabbitt KD. Holes in the reticular lamina after noise exposure: implication for continuing damage in the organ of Corti. Hear Res. 1983;11(1):41-53.

14. Forge A. Outer hair cell loss and supporting cell expansion following chronic gentamicin treatment. Hear Res. 1985;19(2):171-182.

15. Leonova EV, Raphael Y. Organization of cell junctions and cytoskeleton in the reticular lamina in normal and ototoxically damaged organ of Corti. Hear Res. 1997;113(1-2):14-28.

16. Bird JE, Daudet N, Warchol ME, Gale JE. Supporting cells eliminate dying sensory hair cells to maintain epithelial integrity in the avian inner ear. J Neurosci. 2010;30(37):12545-12556.

17. Monzack EL, May LA, Roy S, Gale JE, Cunningham LL. Live imaging the phagocytic activity of inner ear supporting cells in response to hair cell death. Cell Death Differ. 2015;22(12):1995-2005.

18. May LA, et al. Inner ear supporting cells protect hair cells by secreting HSP70.J Clin Invest . 2013;123(8):3577-3587.

19. Mambula SS, Calderwood SK. Heat shock protein 70 is secreted from tumor cells by a nonclassical pathway involving lysosomal endosomes. JImmunol. 2006;177(11):7849-7857.

20. De Maio A. Extracellular Hsp70: export and function. Curr Protein Pept Sci. 2014;15(3):225-231.

21. Kim DK, et al. EVpedia: a community web portal for extracellular vesicles research. Bioinformatics. 2015;31(6):933-939.

22. Colombo M, Raposo G, Théry C. Biogenesis, secretion, and intercellular interactions of exosomes and other extracellular vesicles. Anпи Rev Cell Dev Biol. 2014;30:255-289.

23. Lancaster GI, Febbraio MA. Exosome-dependent trafficking of HSP70: a novel secretory pathway for cellular stress proteins. J Biol Chem. 2005;280(24):23349-23355.

24. Peinado $\mathrm{H}$, et al. Melanoma exosomes educate bone marrow progenitor cells toward a pro-metastatic phenotype through MET. Nat Med. 2012;18(6):883-891.

25. Takeuchi T, et al. Intercellular chaperone transmission via exosomes contributes to maintenance of protein homeostasis at the organismal level. Proc Natl Acad Sci USA. 2015;112(19):E2497-E2506.

26. Lv LH, et al. Anticancer drugs cause release of exosomes with heat shock proteins from human hepatocellular carcinoma cells that elicit effective natural killer cell antitumor responses in vitro. J Biol Chem. 2012;287(19):15874-15885.

27. Clayton A, Turkes A, Navabi H, Mason MD, Tabi Z. Induction of heat shock proteins in B-cell exosomes. J Cell Sci. 2005;118(Pt 16):3631-3638.

28. Filipe V, Hawe A, Jiskoot W. Critical evaluation of nanoparticle tracking analysis (NTA) by NanoSight for the measurement of nanoparticles and protein aggregates. Pharm Res. 2010;27(5):796-810.

29. Théry C, Amigorena S, Raposo G, Clayton A. Isolation and characterization of exosomes from cel culture supernatants and biological fluids. Curr Protoc Cell Biol. 2006; Chapter 3:Unit 3.22.

30. Gardiner C, et al. Techniques used for the isolation and characterization of extracellular vesicles: results of a worldwide survey. J Extracell Vesicles. 2016;5:32945.

31. Wu Y, Deng W, Klinke DJ. Exosomes: improved methods to characterize their morphology, RNA content, and surface protein biomarkers. Analyst. 2015;140(19):6631-6642.

32. Raposo G, Stoorvogel W. Extracellular vesicles: exosomes, microvesicles, and friends. JCell Biol. 2013;200(4):373-383.

33. Nordin JZ, et al. Ultrafiltration with size-exclusion liquid chromatography for high yield isolation of extracellular vesicles preserving intact biophysical and functional properties. Nanomedicine. 2015;11(4):879-883.

34. Kowal J, Tkach M, Théry C. Biogenesis and secretion of exosomes. Curr Opin Cell Biol. 2014;29:116-125.

35. Mi H, et al. PANTHER version 11: expanded annotation data from Gene Ontology and reactome pathways, and data analysis tool enhancements. Nucleic Acids Res. 2017;45(D1):D183-D189.

36. Thomas PD, et al. PANTHER: a library of protein families and subfamilies indexed by function. Genome Res. 2003;13(9):2129-2141.

37. Supek F, Bošnjak M, Škunca N, Šmuc T. REVIGO summarizes and visualizes long lists of gene ontology terms. PLoS ONE. 2011;6(7):e21800.

38. Zutter MM. Integrin-mediated adhesion: tipping the balance between chemosensitivity and chemoresistance. Adv Exp Med Biol. 2007;608:87-100.

39. Hynes RO. Integrins: bidirectional, allosteric signaling machines. Cell. 2002;110(6):673-687.

40. Hoshino A, et al. Tumour exosome integrins determine organotropic metastasis. Nature. 2015;527(7578):329-335.

41. Trajkovic K, et al. Ceramide triggers budding of exosome vesicles into multivesicular endosomes. Science. 2008;319(5867):1244-1247.

42. Hannun YA. Functions of ceramide in coordinating cellular responses to stress. Science. 1996;274(5294):1855-1859.

43. Arenz C, Giannis A. Synthesis of the first selective irreversible inhibitor of neutral sphingomyelinase. Angew Chem Int Ed Engl. 2000;39(8):1440-1442.

44. Muzumdar MD, Tasic B, Miyamichi K, Li L, Luo L. A global double-fluorescent Cre reporter mouse. Genesis. 2007;45(9):593-605.

45. Heusermann W, et al. Exosomes surf on filopodia to enter cells at endocytic hot spots, traffic within endosomes, and are targeted to the ER. JCell Biol. 2016;213(2):173-184.

46. Lai CP, et al. Visualization and tracking of tumour extracellular vesicle delivery and RNA translation using multiplexed reporters. Nat Commun.
2015;6:7029.

47. Gastpar R, et al. Heat shock protein 70 surface-positive tumor exosomes stimulate migratory and cytolytic activity of natural killer cells. Cancer Res. 2005;65(12):5238-5247.

48. Vega VL, et al. Hsp70 translocates into the plasma membrane after stress and is released into the extracellular environment in a membrane-associated form that activates macrophages. JImmunol. 2008;180(6):4299-4307.

49. Cho JA, Lee YS, Kim SH, Ko JK, Kim CW. MHC independent anti-tumor immune responses induced by Hsp70-enriched exosomes generate tumor regression in murine models. Cancer Lett. 2009;275(2):256-265.

50. Chalmin F, et al. Membrane-associated Hsp72 from tumor-derived exosomes mediates STAT3-dependent immunosuppressive function of mouse and human myeloid-derived suppressor cells. JClin Invest. 2010;120 (2):457-471.

51. Roué G, Pichereau V, Lincet H, Colomer D, Sola B. Cyclin D1 mediates resistance to apoptosis through upregulation of molecular chaperones and consequent redistribution of cell death regulators. Oncogene. 2008;27(36):4909-4920.

52. Li CJ, Ning W, Matthay MA, Feghali-Bostwick CA, Choi AM. MAPK pathway mediates EGR1-HSP70-dependent cigarette smoke-induced chemokine production. Am J Physiol Lung Cell Mol Physiol. 2007;292(5):L1297-L1303.

53. Jheng HF, et al. Albumin stimulates renal tubular inflammation through an HSP70-TLR4 axis in mice with early diabetic nephropathy. Dis Model Mech. 2015;8(10):1311-1321.

54. Vabulas RM, Ahmad-Nejad P, Ghose S, Kirschning CJ, Issels RD, Wagner H. HSP7O as endogenous stimulus of the Toll/interleukin-1 receptor signal pathway. J Biol Chem. 2002;277(17):15107-15112.

55. Luong M, et al. Stimulation of TLR4 by recombinant HSP7O requires structural integrity of the HSP70 protein itself. J Inflamm (Lond). 2012;9:11.

56. Asea A, et al. Novel signal transduction pathway utilized by extracellular HSP70: role of tolllike receptor (TLR) 2 and TLR4.J Biol Chem. 2002;277(17):15028-15034.

57. de Oliveira AA, Faustino J, de Lima ME, Menezes $\mathrm{R}$, Nunes KP. Unveiling the interplay between the TLR4/MD2 complex and HSP7O in the human cardiovascular system: a computational approach. Int J Mol Sci. 2019;20(13):E3121.

58. Vicencio JM, et al. Plasma exosomes protect the myocardium from ischemia-reperfusion injury. J Am Coll Cardiol. 2015;65(15):1525-1536.

59. Zhang Y, Zhang X, Shan P, Hunt CR, Pandita TK, Lee PJ. A protective Hsp70-TLR4 pathway in lethal oxidant lung injury. J Immunol. 2013;191(3):1393-1403.

60. Chen T, Guo J, Yang M, Zhu X, Cao X. Chemokine-containing exosomes are released from heat-stressed tumor cells via lipid raft-dependent pathway and act as efficient tumor vaccine. JImmunol. 2011;186(4):2219-2228.

61. EL Andaloussi S, Mäger I, Breakefield XO, Wood MJ. Extracellular vesicles: biology and emerging therapeutic opportunities. Nat Rev Drug Discov. 2013;12(5):347-357.

62. De Maio A, Vazquez D. Extracellular heat shock 
proteins: a new location, a new function. Shock. 2013;40(4):239-246.

63. Elsner L, et al. The heat shock protein HSP7O promotes mouse NK cell activity against tumors that express inducible NKG2D ligands. J Immunol. 2007;179(8):5523-5533.

64. Takeuchi $\mathrm{T}$, et al. Intercellular chaperone transmission via exosomes contributes to maintenance of protein homeostasis at the organismal level. Proc Natl Acad Sci USA. 2015;112(19):E2497-E2506.

65. Gobbo J, et al. Restoring anticancer immune response by targeting tumor-derived exosomes with a HSP7O peptide aptamer. J Natl Cancer Inst. 2016;108(3):djv330.

66. Davidson SM, et al. Cardioprotection mediated by exosomes is impaired in the setting of type II diabetes but can be rescued by the use of non-diabetic exosomes in vitro. J Cell Mol Med. 2018;22(1):141-151.

67. Furness DN. Molecular basis of hair cell loss. Cell Tissue Res. 2015;361(1):387-399.

68. Lanvers-Kaminsky C, Zehnhoff-Dinnesen AA, Parfitt R, Ciarimboli G. Drug-induced ototoxicity: mechanisms, pharmacogenetics, and protective strategies. Clin Pharmacol Ther. 2017;101(4):491-500.

69. Batrakova EV, Kim MS. Using exosomes, naturallyequipped nanocarriers, for drug delivery. JControl Release. 2015;219:396-405.

70. Bunggulawa EJ, et al. Recent advancements in the use of exosomes as drug delivery systems. Journal of Nanobiotechnology. 2018;16(1):81.

71. Conlan RS, Pisano S, Oliveira MI, Ferrari M, Mendes Pinto I. Exosomes as Reconfigurable Therapeutic Systems. Trends Mol Med.
2017;23(7):636-650.

72. Murphy DE, et al. Extracellular vesicle-based therapeutics: natural versus engineered targeting and trafficking. Exp Mol Med. 2019;51(3):32.

73. van Dommelen SM, et al. Microvesicles and exosomes: opportunities for cell-derived membrane vesicles in drug delivery. J Control Release. 2012;161(2):635-644.

74. Neng L, Zhang F, Kachelmeier A, Shi X. Endothelial cell, pericyte, and perivascular resident macrophage-type melanocyte interactions regulate cochlear intrastrial fluid-blood barrier permeability. J Assoc Res Otolaryngol. 2013;14(2):175-185.

75. Zhang W, et al. Perivascular-resident macrophage-like melanocytes in the inner ear are essential for the integrity of the intrastrial fluid-blood barrier. Proc Natl Acad Sci USA. 2012;109(26):10388-10393.

76. Alvarez-Erviti L, Seow Y, Yin H, Betts C, Lakhal S, Wood MJ. Delivery of siRNA to the mouse brain by systemic injection of targeted exosomes. Nat Biotechnol. 2011;29(4):341-345.

77. György B, et al. Rescue of hearing by gene delivery to inner-ear hair cells using exosome-associated AAV. Mol Ther. 2017;25(2):379-391.

78. Matern M, et al. Gfi1 ${ }^{\text {Cre }}$ mice have early onset progressive hearing loss and induce recombination in numerous inner ear non-hair cells. Sci Rep. 2017;7:42079.

79. Heng JS, et al. Hypoxia tolerance in the Norrin-deficient retina and the chronically hypoxic brain studied at single-cell resolution. Proc Natl Acad Sci USA. 2019;116(18):9103-9114.

80. Stone JS, Wisner SR, Bucks SA, Mellado Lagarde MM, Cox BC. Characterization of adult vestib- ular organs in 11 CreER mouse lines. J Assoc Res Otolaryngol. 2018;19(4):381-399.

81. Yang H, Xie X, Deng M, Chen X, Gan L. Generation and characterization of Atoh1-Cre knock-in mouse line. Genesis. 2010;48(6):407-413.

82. Cunningham LL. The adult mouse utricle as an in vitro preparation for studies of ototoxicdrug-induced sensory hair cell death. Brain Res. 2006;1091(1):277-281.

83. Brandon CS, Voelkel-Johnson C, May LA, Cunningham LL. Dissection of adult mouse utricle and adenovirus-mediated supporting-cell infection. JVis Exp. 2012;(61):3734.

84. Shelke GV, Lässer C, Gho YS, Lötvall J. Importance of exosome depletion protocols to eliminate functional and RNA-containing extracellular vesicles from fetal bovine serum. J Extracell Vesicles. 2014;3:24783.

85. Schindelin J, Rueden CT, Hiner MC, Eliceiri KW. The Image e ecosystem: An open platform for biomedical image analysis. Mol Reprod Dev. 2015;82(7-8):518-529.

86. Lin Y, et al. Sodium laurate, a novel protease- and mass spectrometry-compatible detergent for mass spectrometry-based membrane proteomics. PLoS ONE. 2013;8(3):e59779.

87. Rappsilber J, Mann M, Ishihama Y. Protocol for micro-purification, enrichment, pre-fractionation and storage of peptides for proteomics using StageTips. Nat Protoc. 2007;2(8):1896-1906.

88. Kaufmann SH. Reutilization of immunoblots after chemiluminescent detection. Anal Biochem. 2001;296(2):283-286.

89. Holm S. A simple sequentially rejective multiple test procedure. Scandinavian Journal of Statistics. 1979;6(2):65-70. 